\title{
Understanding and optimizing microstrip patch antenna cross polarization radiation on element level for demanding phased array antennas in weather radar applications
}

\author{
D. Vollbracht \\ Faculty of Electrical Engineering and Information Technology, Technische Universität Chemnitz, 09126 Chemnitz, Germany \\ Correspondence to: D. Vollbracht (dennis-vollbracht@web.de) \\ Received: 21 December 2014 - Revised: 6 March 2015 - Accepted: 16 March 2015 - Published: 3 November 2015
}

\begin{abstract}
The antenna cross polarization suppression (CPS) is of significant importance for the accurate calculation of polarimetric weather radar moments. State-of-the-art reflector antennas fulfill these requirements, but phased array antennas are changing their CPS during the main beam shift, off-broadside direction. Since the cross polarization (x-pol) of the array pattern is affected by the x-pol element factor, the single antenna element should be designed for maximum CPS, not only at broadside, but also for the complete angular electronic scan (e-scan) range of the phased array antenna main beam positions.

Different methods for reducing the x-pol radiation from microstrip patch antenna elements, available from literature sources, are discussed and summarized. The potential x-pol sources from probe fed microstrip patch antennas are investigated. Due to the lack of literature references, circular and square shaped X-Band radiators are compared in their X-pol performance and the microstrip patch antenna size variation was analyzed for improved x-pol pattern.

Furthermore, the most promising technique for the reduction of x-pol radiation, namely "differential feeding with two RF signals $180^{\circ}$ out of phase", is compared to single fed patch antennas and thoroughly investigated for phased array applications with simulation results from CST MICROWAVE STUDIO (CST MWS). A new explanation for the excellent port isolation of dual linear polarized and differential fed patch antennas is given graphically. The antenna radiation pattern from single fed and differential fed microstrip patch antennas are analyzed and the shapes of the x-pol patterns are discussed with the well-known cavity model. Moreover, two new visual based electromagnetic approaches for the explanation of the $\mathrm{x}-$ pol generation will be given: the field line approach and the surface current distribution approach
\end{abstract}

provide new insight in understanding the generation of $\mathrm{x}$-pol component in microstrip patch antenna radiation patterns.

\section{Introduction}

Dual polarized phased array antennas for weather radar application in dense radar networks are currently under discussion (Vollbracht, 2014). Such antennas promise even faster three dimensional volume scanning compared to state-of-theart high power radar systems with reflector antennas and their twin axis mechanical drives. The change from parabolic reflector antennas with fixed beamwidth and gain values to phased array antennas with inherent scan angle dependent $\mathrm{co} / \mathrm{x}$-pol patterns is highly challenging.

Weather radar systems with dual polarization capability observe echoes backscattered by hydrometeors from illuminated volumes to classify the precipitation, primarily with horizontally and vertically polarized electromagnetic waves. For accurate polarimetric echo measurements and their interpretation, the antenna CPS performances for both polarization planes are of significant importance. Depending on the CPS, the estimation of the Differential Reflectivity (ZDR) and the Linear Depolarization Ratio (LDR) can be biased. High-quality reflector antennas provide CPS values of up to $-30 \mathrm{~dB}$ in S- C- X- Band, within the half power beamwidth. A new phased array weather radar antenna should provide the same CPS on broadside, and even more challenging, the same CPS over the complete e-scan range. The e-scan ranges stated from (Vollbracht, 2014) are $120^{\circ}$ azimuth and $30^{\circ}$ elevation for one planar phased array antenna panel. One 3-D volume radar scanner presented from (Vollbracht, 2014) 
would be equipped with three of such planar phased array antennas to achieve $360^{\circ}$ azimuth volume scanning.

From phased array antenna theory the co- and x-pol antenna radiation pattern can be calculated with the multiplication law that gives the overall antenna pattern as product of the element pattern (EP) and the array factor (AF). Consequently special attention should be paid to the design of the single antenna element and its EP in order to avoid unwanted $\mathrm{x}$-pol radiation right from the very beginning.

Antenna manufacturers typically provide only $\mathrm{E}$ and $\mathrm{H} \mathrm{Cut}$ radiation pattern. For phased array weather radar applications this amount of information is inadequate. If the beam were to be pointed off- broadside- axis, the CPS would be much lower. As a consequence, all antennas for phased array weather applications should be analyzed also in the $45^{\circ} / 135^{\circ}$ Cut planes, since the highest $\mathrm{x}$-pol radiation may be expected in this region. A detailed $\mathrm{x}$-pol investigation can only be performed by the 3-D analysis of the complete upper hemisphere of the microstrip patch element pattern.

Dual polarized microstrip patch antennas are suitable candidates for phased array weather radar applications. These antennas are fabricated easily at low cost and have known radiation characteristics. On single antenna element level, the different $\mathrm{x}$-pol reduction methods and their performances are now investigated and discussed. Finally new visual based electromagnetic approaches for the explanation of the x-pol generation are introduced.

\section{X-pol suppression techniques on element level known from literature}

Only a few suppression techniques on antenna element level have been published. The authors from Liang et al. (2004), Fulton and Chappell (2011) and Zhou and Chio (2011) use differential feed excitation with two signals, $180^{\circ}$ out of phase. This method will be explained in detail by the antenna prototype in 2.2 and promises to be a viable solution for phased array weather radar antennas. The defective ground plane solution published in Guha et al. (2005) is useful for single element applications without complex feeding networks and dual polarization capabilities. The radiation from the defective ground area induces amplitude taper disturbances to the array feeding network and would degrade the overall antenna performance. Mohanty and Das (1993) presented a printed thin dielectric substrate with strip grating in front of the radiating antennas for suppressing the x-pol radiation. But strip grating solutions are only useful for single polarization applications. Recently a new investigation to explain $\mathrm{x}$-pol radiation from microstrip antennas was published in Bhardwaj and Rahmat-Samii (2014). Here the $\mathrm{x}$ pol for differential feeding ( $180^{\circ}$ out of phase) and rotational feeding (mirrored antenna elements inside antenna array) are discussed by analyzing the near-field radiation behavior. The major intention of the authors (Bhardwaj and Rahmat-Samii,
2014) is to provide a new understanding of the x-pol radiation generation by observing the near field disturbances. The explanations of the $\mathrm{x}$-pol generation process in Sects. 4.2 and 4.3 can be understood as an additional contribution to the visual based near field approach from Bhardwaj and RahmatSamii (2014), but here with field lines and surface currents. A comparison of x-pol performances of circular and rectangular/square microstrip radiators for dual linear polarization applications was surprisingly not found in available literature. Also no capable literature reference could be found where the patch antenna size variation was analyzed for improved $x-$ pol pattern. Consequently the circular and rectangular shape types and the patch antenna size variation are investigated and compared in Sect. 3.3 and 3.5.

\subsection{Feeding methods for low $\mathrm{x}$-pol radiation in microstrip array design}

There are numerous feeding mechanisms available for microstrip patch antenna elements. Well-known feeding techniques are aperture-coupling, probe-feeding, proximitycoupling and insert-feeding. Aperture-coupled patch antennas are often used to enhance the bandwidth with the radiating slots underneath the patch radiator. To further enhance the bandwidth, a second patch separated with foam material from the first one and with slightly different dimensions and corresponding resonance frequency is implemented. The second patch has also the advantage of reducing the x-pol radiation, since the $\mathrm{x}$-pol radiation generated by the lower patch and the slots are shadowed. The patch antenna design from Liang et al. (2004) shows great CPS performances for horizontal and vertical port of -30 to $-35 \mathrm{~dB}$, respectively. But due to the aperture-coupled radiating slots and the back radiation to the feeding network, the mutual coupling aspect becomes critical for array applications. Further disadvantages are the sensitivity against height tolerances of the foam material and the generation of surface waves due to the radiating slots and the cavities between the foam layers. Moreover, the design presented in Liang et al. (2004) is working with the reflector ground plane technique, which is not useful for array operation with multiple elements and feeding networks between.

Proximity coupled patches have similar disadvantages for array configurations as aperture-coupled antennas. The back radiation close to the feeding networks for two polarization planes is undesired in array configurations and the port isolation would become insufficient. Insert fed microstrip patches have their feeding networks on the same layer and unwanted $\mathrm{x}$-pol radiation is generated. In keeping with these observations, insert fed and proximity coupled patch antennas are not suitable for phased arrays with high x-pol requirements.

Probe fed microstrip patch antennas are suitable candidates for the phased array weather radar application with high x-pol requirements. The possibility to drive the single patch antenna element with $180^{\circ}$ out of phase and the low 


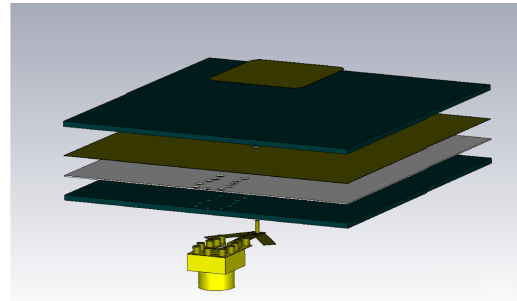

a) Single probe- fed patch

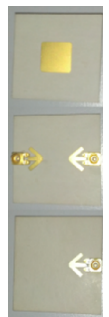

b) Manufactured patches

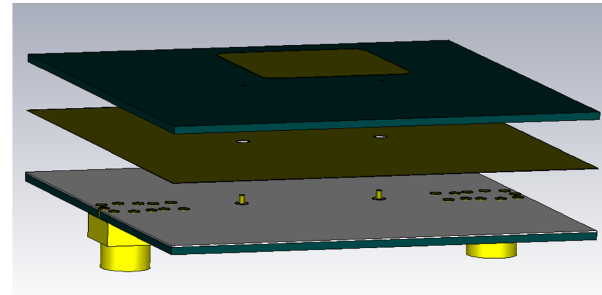

c) Differential probe- fed patch

Figure 1. Layer set-up of squared microstrip patch antenna design.

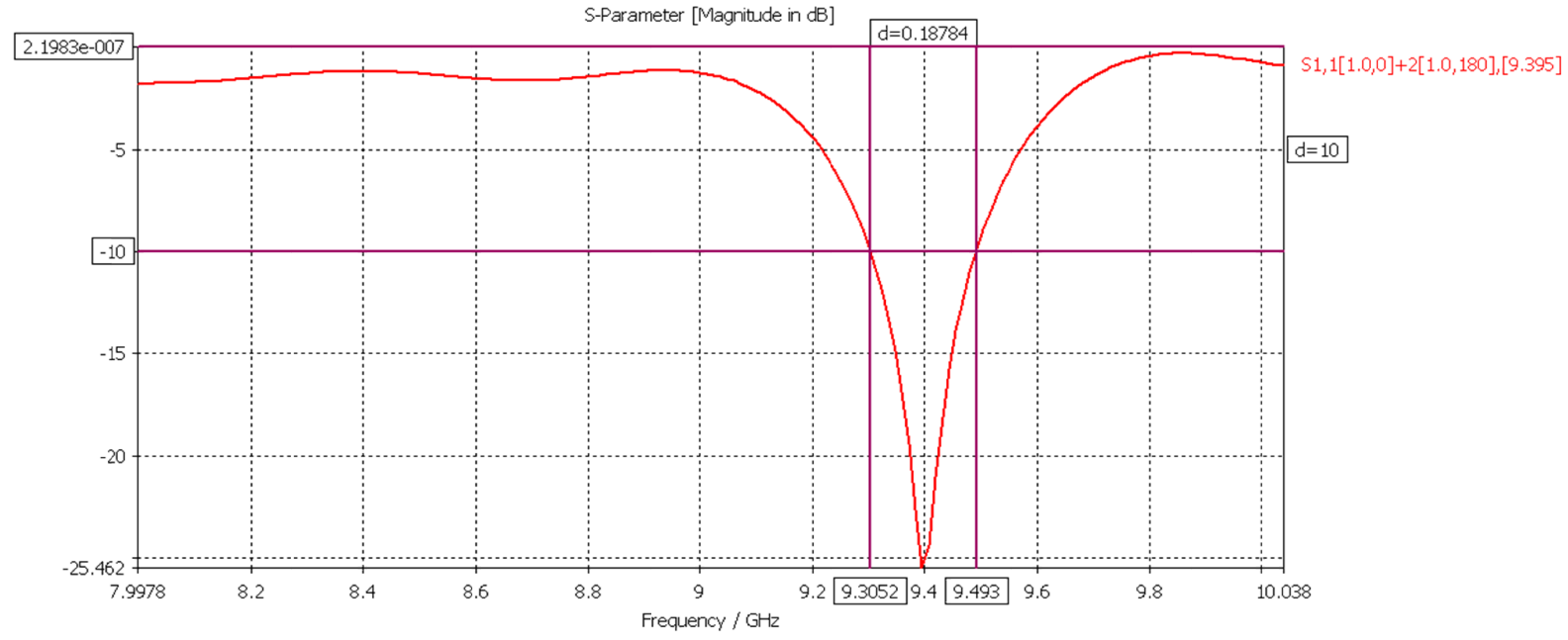

Figure 2. S11 resonating at $9.395 \mathrm{GHz}$ with VSWR $1: 2$ Bandwidth of $188 \mathrm{MHz}$.

back radiation due to the small feed via holes through the shielding ground plane generates only small $\mathrm{x}$-pol contribution.

\subsection{Thorough analysis of the "differential feeding technique" for phased array weather radar applications}

One valuable technique to reduce the $\mathrm{x}$-pol radiation of microstrip antennas was presented by Liang et al. (2004) and Fulton and Chappell (2011) and is called "differential feeding". Differential feeding can be established with two RF drive signals exactly $180^{\circ}$ out of phase. To demonstrate and to analyze the advantages of differentially fed microstrip patch antennas for phased array weather radar applications two probe fed antenna designs with single and differential phase feeding are compared. It will be further explained with these antenna prototypes how the $\mathrm{x}$-pol radiation is generated from surface currents and field line distributions.

The square probe fed microstrip patch antennas were designed and simulated with CST MWS. The patch antennas are consisting of two RO6002 substrates (dark green in Fig. 1) with $\varepsilon_{r}=2.94,508 \mu \mathrm{m}$ height and excellent dissipation factor $\tan \delta=0.0012$ for adequate antenna efficiency performances. The grey layer represents in Fig. 1a and $\mathrm{b}$ the RO4403 prepreg with $\varepsilon_{r}=3.17$ and $100 \mu \mathrm{m}$ height. The RO4403 prepreg will be used to combine the two RO6002 laminates during the compression process. For suppressing unwanted surface currents or propagation channels within the substrate right from the design beginning, this prepreg shows only small $\varepsilon_{r}$ differences compared to RO6002. The copper has a thickness of $35 \mu \mathrm{m}$ and the diameter of the isolation circle inside the shielding ground for the probe feed transition is $0.91 \mathrm{~mm}$. The radiating square patches with $10.55 \mathrm{~mm}$ edge length, pin-via diameter of 0.4 and $0.416 \mathrm{~mm}$ pin- via to edge- distance are designed for a resonance frequency of $9.395 \mathrm{GHz}$ (see Fig. 2).

Both antennas from Fig. 1 are sharing the almost same design parameters. The only differences are the additional port in Fig. 1c with $180^{\circ}$ out of phase excitation and the width and length of the matching circuit (arrow) close to the feed. The Rosenberger 3-D connector model was completely integrated in the CST MWS full wave simulation and impedance matched with GND vias and microstrip impedance transformer to the $50 \Omega$ line impedance. The microstrip stubs in parallel to the probe feed are used for matching the impedance of the patch antenna. In Fig. 1b the manufactured patches are visualized. 

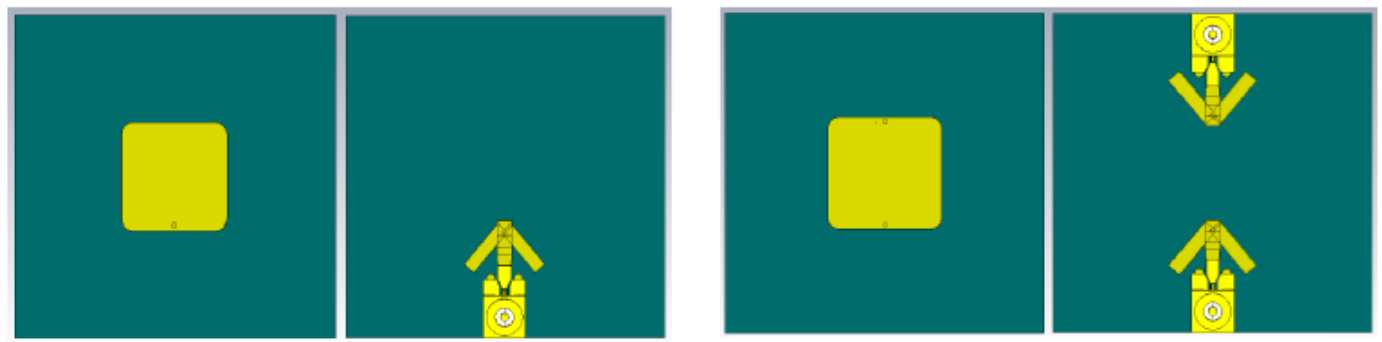

6

a-1) Single probe- fed patch

b-1)Differential probe- fed patch

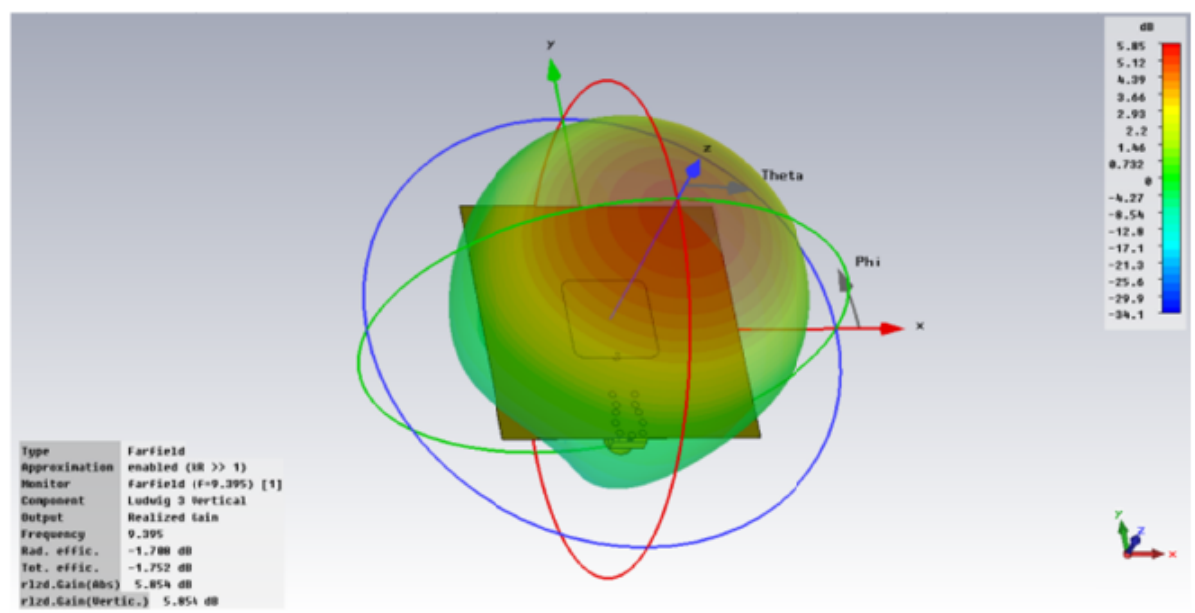

a-2) Single probe- fed co-pol 3D Pattern

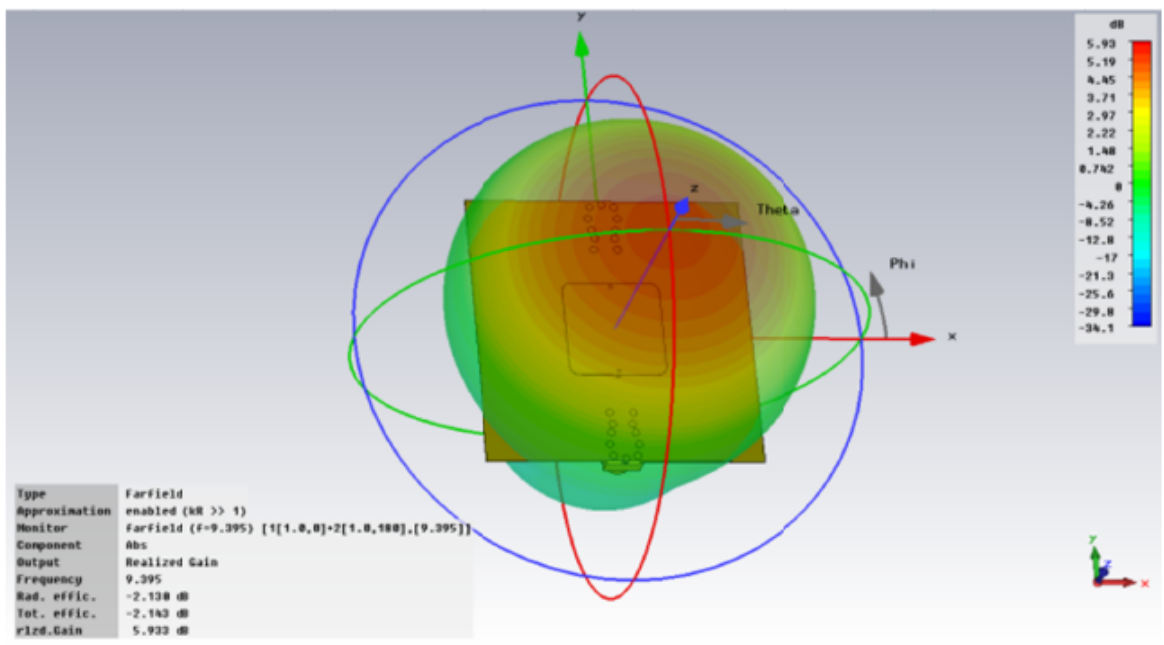

b-2) Differential probe- fed co- pol 3D Pattern

Figure 3.

Figure 2 shows the S11 of the matched connector port with patch antenna. The VSWR $1: 2$ bandwidth of $188 \mathrm{MHz}$ was simulated.

Figure 3 compares the co/x-pol pattern without (Fig. 3a) and with (Fig. 3b) the differential feeding technique. The radiation patterns are plotted with Ludwig's third definition of
X-pol (Ludwig, 1973). Note that the reference parameter "realized gain" in $\mathrm{dBi}$ also considers the impedance mismatch losses for the gain calculations. In the left row of Fig. 3 the single probe fed patch antenna radiation patterns are shown together with the co-pol "realized gain" of $5.85 \mathrm{dBi}$. The right row of Fig. 3 displays the radiation patterns of the dif- 


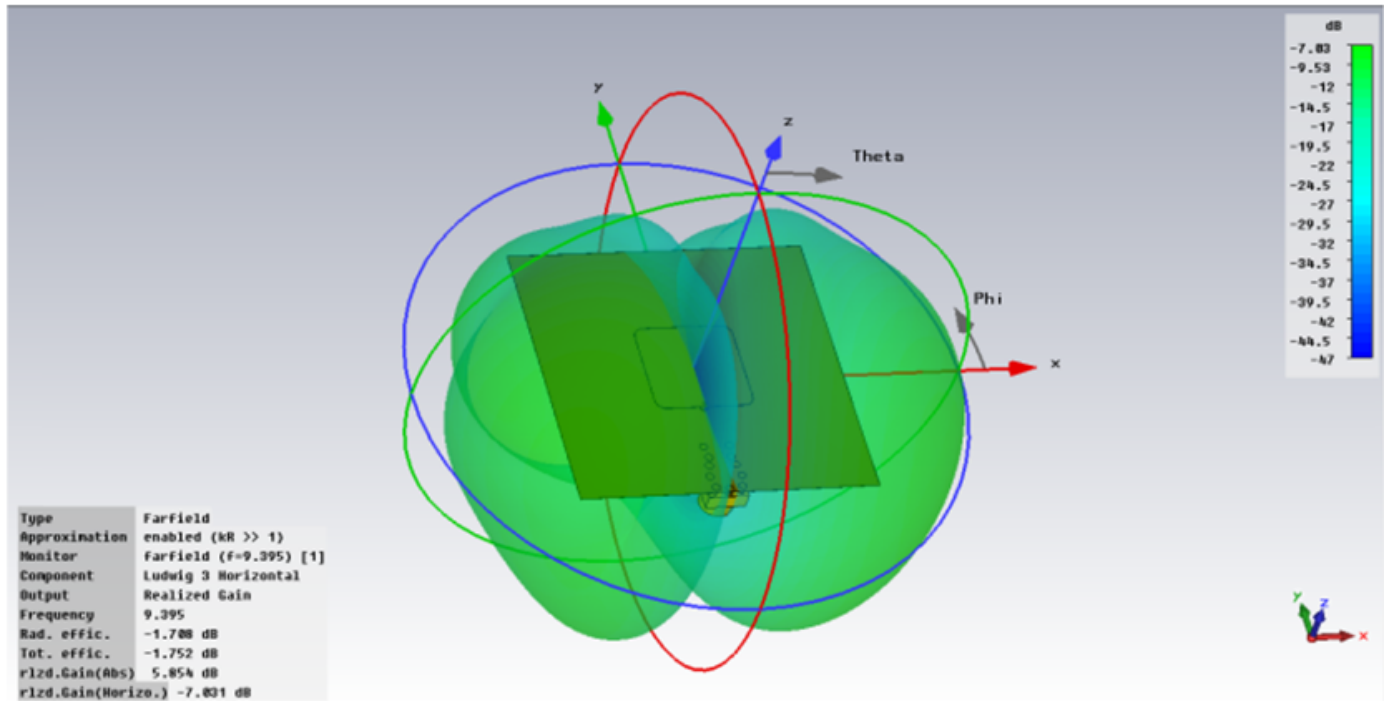

a-3) Single probe- fed $x-p o l$ 3D Pattern

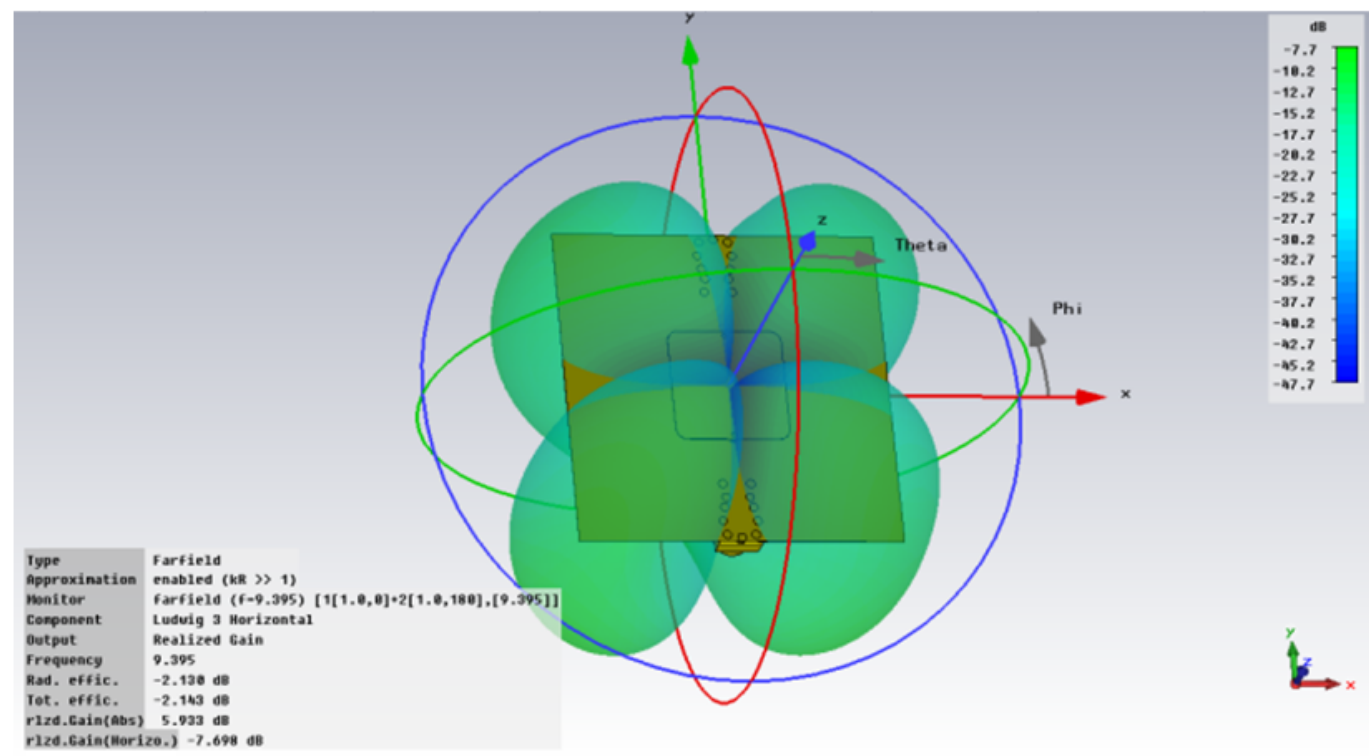

b-3) Differential probe- fed x-pol 3D Pattern

Figure 3.

ferential probe fed patch antenna with $180^{\circ}$ out of phase feeding and the co-pol "realized gain" of $5.93 \mathrm{dBi}$.

The x-pol pattern in Fig. 3a-3 shows two maxima and one null in the E Cut. The radiating edge close to the feed contributes the highest $\mathrm{x}$-pol radiation. The opposite to feed located radiating edge adds much lower $\mathrm{x}$-pol radiation.

By further analyzing the $\mathrm{x}$-pol pattern it is obvious that the $\mathrm{E}\left(\Phi=90^{\circ}\right)$ and $\mathrm{H}$ Cut $\left(\Phi=0^{\circ}\right)$, typically provided by antenna manufacturer, would not show the maximum x-pol radiation intensities from the upper far field hemisphere. The
E Cut CPS would show almost perfect results and the $\mathrm{H} \mathrm{Cut}$ would exhibit a high, but unrealistic x-pol contribution from the 3-D antenna radiation pattern. Only the dedicated $45^{\circ}$ Cut would show the real antenna performance in CPS. For this reason, it is recommended to use $45^{\circ}$ Cuts in any CPS discussions for patch antennas when the integrated CPS is important. Figure 4 shows the comparison of the single and differential probe fed patch antenna CPS performance using the recommended $45^{\circ}$ Cuts. 


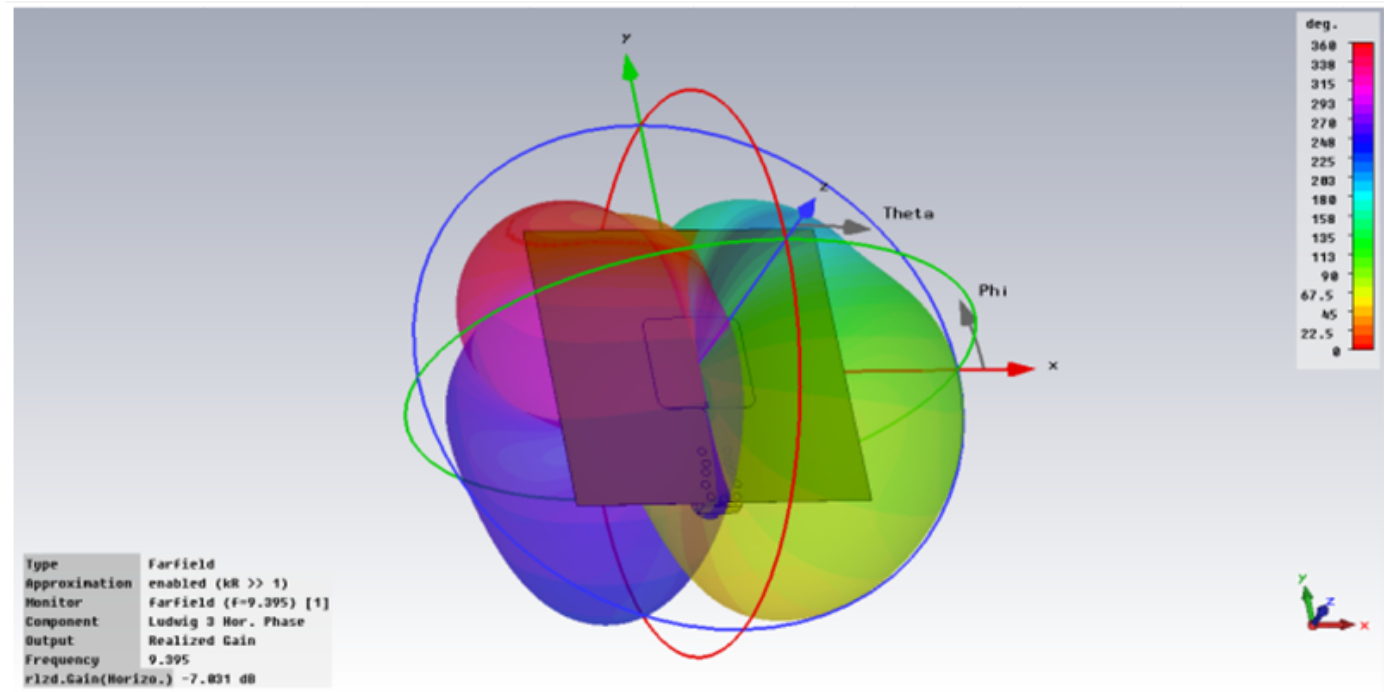

a-4) Single probe- fed phase x-pol 3D Pattern

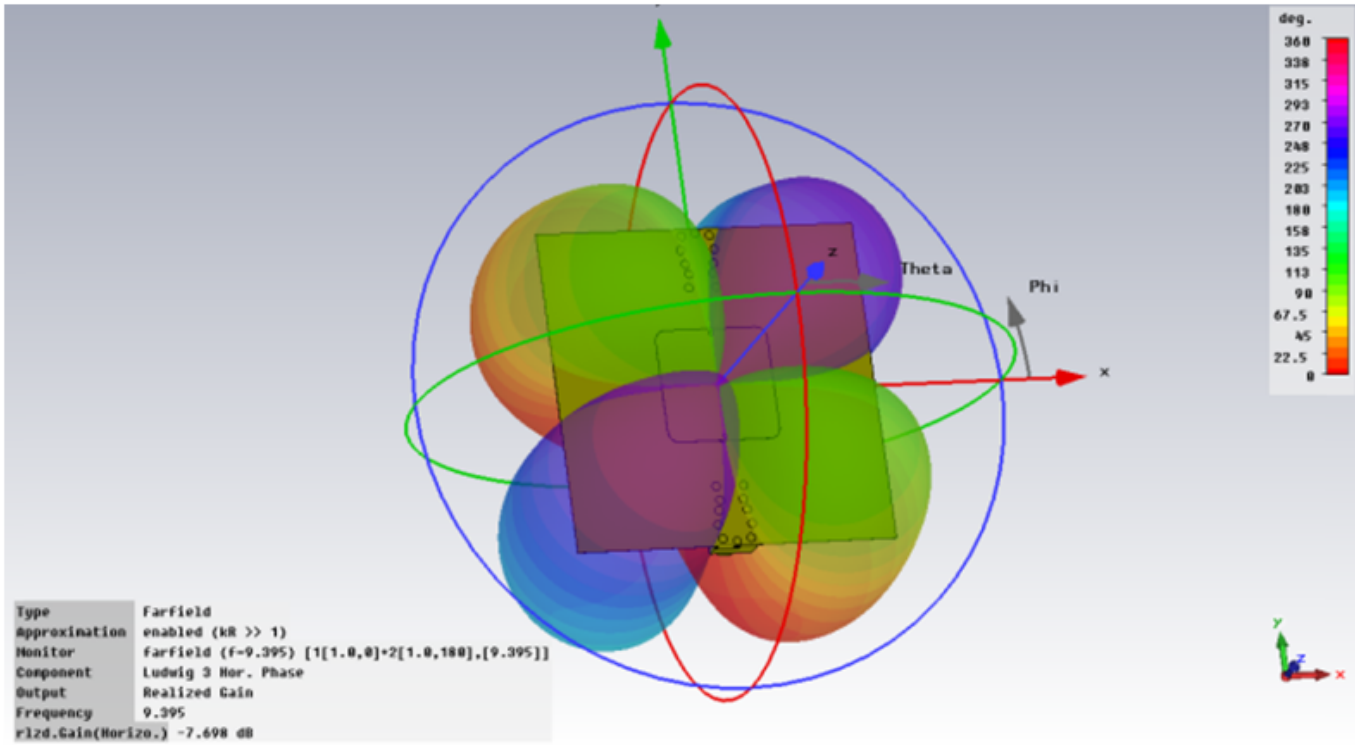

b-4) Differential probe- fed phase x-pol 3D Pattern

Figure 3. Comparison of co/x-pol patterns (a) without and (b) with differential feeding.

The differences in CPS performances are clearly identifiable by the plots in Fig. 4. The co- and x-pol plots from Fig. 4 are generated by the $\Phi=45^{\circ}$ Cuts from Fig. 3a-2, b-2, a-3 and b-3 respectively. Typically phased array antennas are providing azimuth e-scan ranges from +60 to $-60^{\circ}$. Within this angular scan range the integrated CPS should be as low as possible. An integrated CPS of $14.2 \mathrm{~dB}$ for differential fed patch antennas can be achieved at $\pm 60^{\circ} \mathrm{AZ}$ posi- tion, compared to only $6.6 \mathrm{~dB}$ at $-60^{\circ} \mathrm{AZ}$ for the single fed patch antenna. The $3 \mathrm{~dB}$ half power beamwidth highlighted with the ellipse on the center top of Fig. 4 shows another advantage of the differential fed antenna for phased array applications: the beamwidth is quiet symmetric and the phased array scan gain loss with respect to the element pattern would also be symmetric for positive and negative scan angles. The single fed antenna shows a slight shift of the beam maxi- 


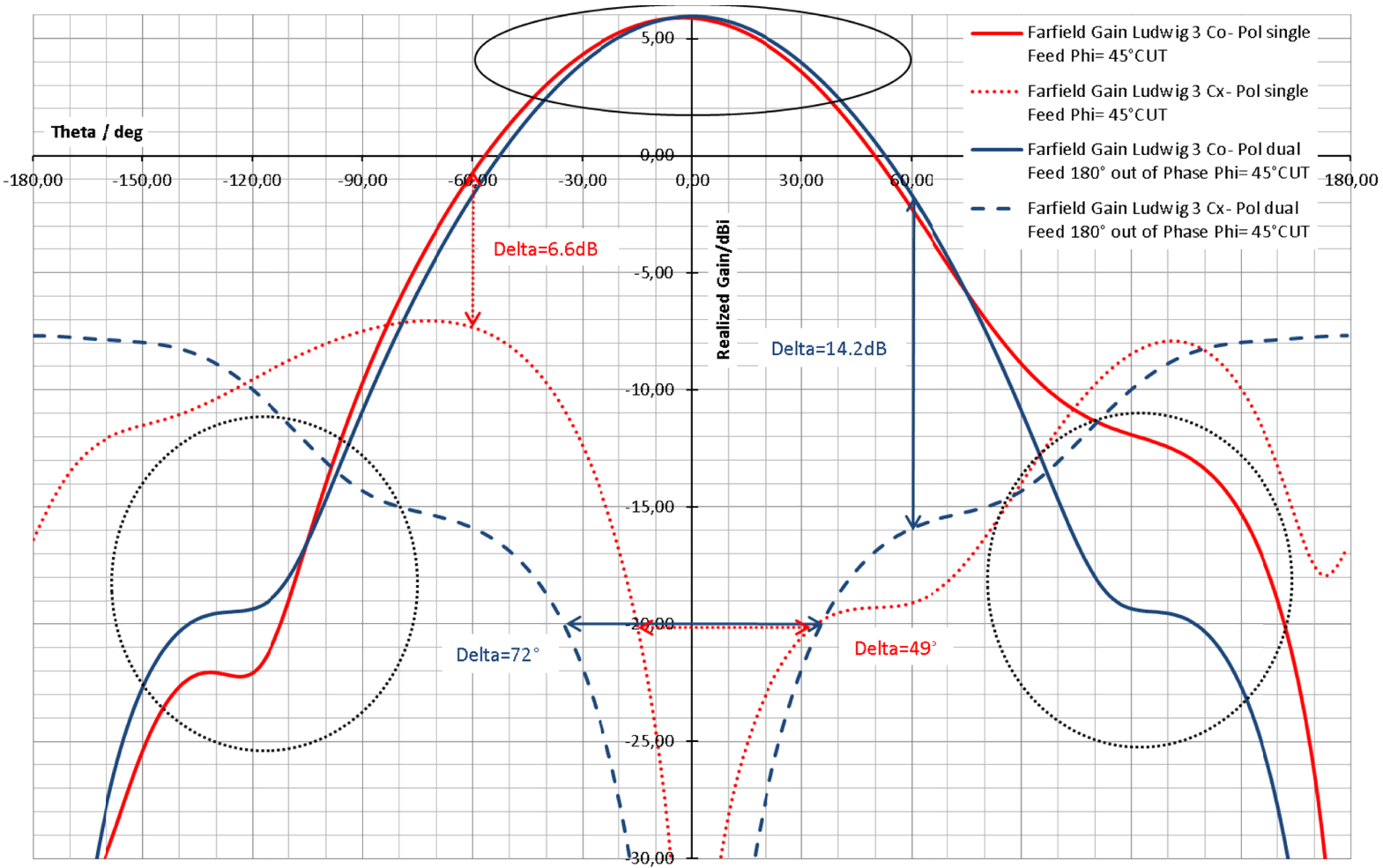

Figure 4. Comparison of single (red) and differential (blue) fed patch antenna CPS performances at $45^{\circ}$ CUT. The dashed curves are representing the gain plots of the $\mathrm{x}$-pol radiation patterns.

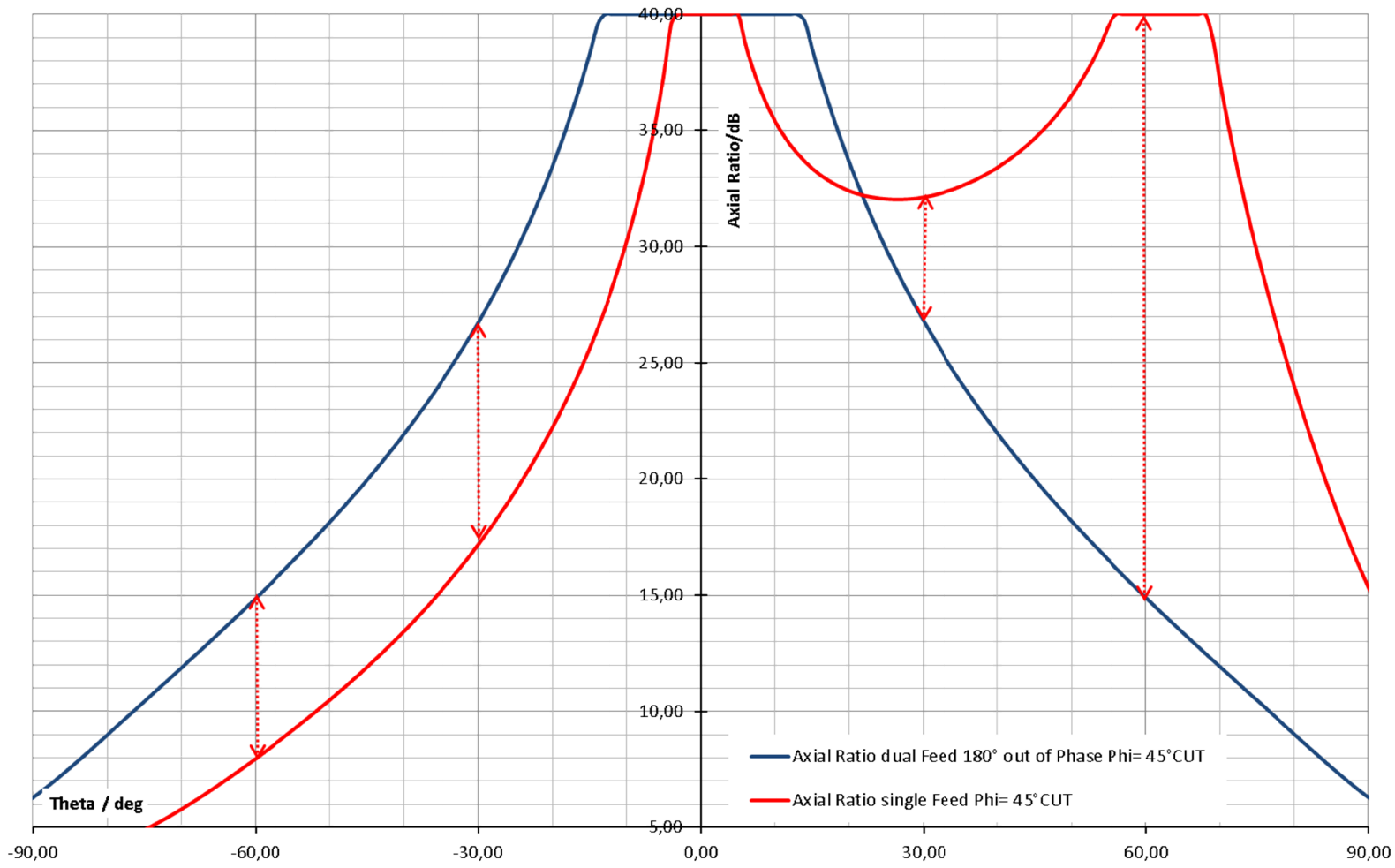

Figure 5. Comparison of single (red) and differential (blue) fed patch antenna axial rations at $45^{\circ}$ CUT. 


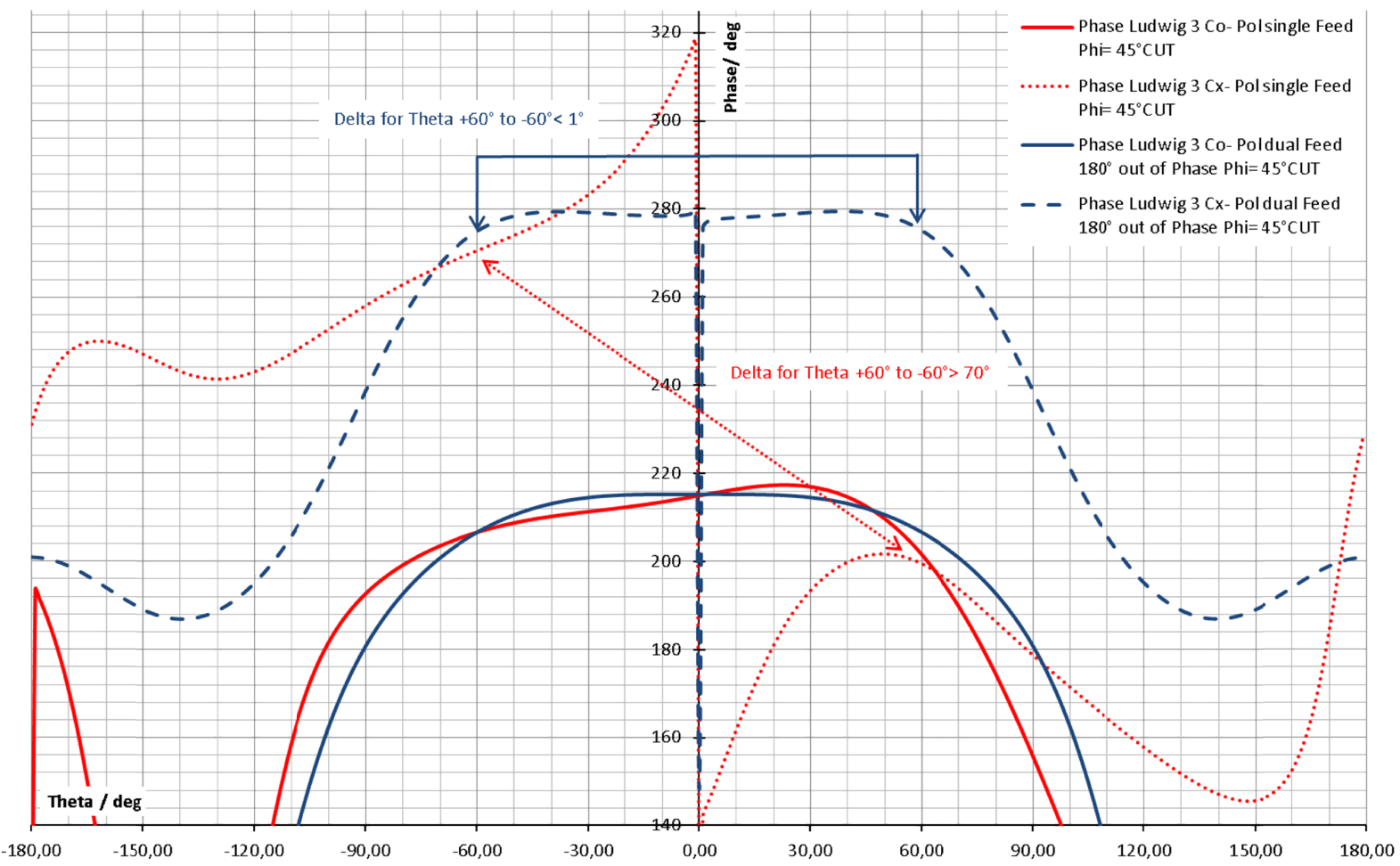

Figure 6. Comparison of Co and X-pol Phase plots from single (red) and differential (blue) fed patch antennas. The dashed curves are representing the phase plots of the $\mathrm{x}$-pol radiation patterns.

mum in negative Theta direction and an asymmetric beam shape. This can be associated to the single feed configuration and the fact that stronger electromagnetic fields are radiated from the radiating edge located close to the feed. The black circles at $\Theta= \pm 120^{\circ}$ indicate back radiated energy and here again the differential fed antenna back radiation is symmetric and would induce the same amount of energy in both backward directions. The mutual coupling would be equal to the neighbor elements compared to the asymmetric back radiation from single fed antennas. Furthermore, the x-pol free area below the main beam at $-20 \mathrm{dBi}$ is much broader for differential feeds with $72^{\circ}$ azimuth range in contrast to only $49^{\circ}$ azimuth range for the single feed configuration. Figure 5 compares the antenna axial ratios of the antennas. CST MWS is following the IEEE-Standard for the calculation of the axial ratio shown in Fig. 5. The axial ratio is the ratio of the major axis to the minor axis of the polarization ellipse. $\boldsymbol{E}_{1}$ represents the horizontal polarized field vector and $\boldsymbol{E}_{2}$ the vertical polarized field vector. It is calculated as follows:

$\mathrm{AR}=\sqrt{\frac{\left|\boldsymbol{E}_{1}^{2}\right|+\left|\boldsymbol{E}_{2}^{2}\right|+\left|\boldsymbol{E}_{1}^{2}+\boldsymbol{E}_{2}^{2}\right|}{\left|\boldsymbol{E}_{1}^{2}\right|+\left|\boldsymbol{E}_{2}^{2}\right|-\left|\boldsymbol{E}_{1}^{2}+\boldsymbol{E}_{2}^{2}\right|}}$.

The axial ratio for the differential feed patch is again symmetric and shows better results for negative and positive scan angles of up to $\Theta=25^{\circ}$. For negative angles the differential feed shows a better axial ratio with smaller decay per degree. The single feed antenna shows for $\Theta>+25^{\circ}$ better results than the differential feed antenna, but when considering all other disadvantages mentioned before this fact is negligible. It should also be noted here that an asymmetric axial ratio induces non- consistent scan angle biases for polarimetric phased array weather radar measurements.

The x-pol phases from Fig. 6 are generated from the $\Phi=45^{\circ}$ Cut of Fig. 3a-4 and b-4 respectively. The co-pol phase plots are not visualized in 3-D. It can be recognized that the co- and $\mathrm{x}$-pol phase of the differential fed antenna is very symmetrical distributed, especially in the desired scan range of $\pm 60^{\circ}$. The phase is identical over diagonal plane (see Fig. 3b-4). The co- and $x-$ phases of the single fed antenna are asymmetric and the maxima of $\mathrm{x}$-pol phases on diagonal planes are more than $70^{\circ}$ out of phase. By analyzing the $\Phi=135^{\circ}$ and $\Phi=45^{\circ}$ Cuts from Fig. 7, a phase difference of exactly $180^{\circ}$ can be observed for the complete $\Theta$ range. Each point is exactly $180^{\circ}$ out of phase by comparing both diagonal planes. This predictable diagonal phase distribution can be used for array configurations where the neighboring elements $\mathrm{x}$-pol contributions cancel each other in far field.

Furthermore, the two designs from Fig. 3 are redesigned for dual polarization mode to be comparable in their polarimetric port isolation characteristic. State-of-the-art weather 


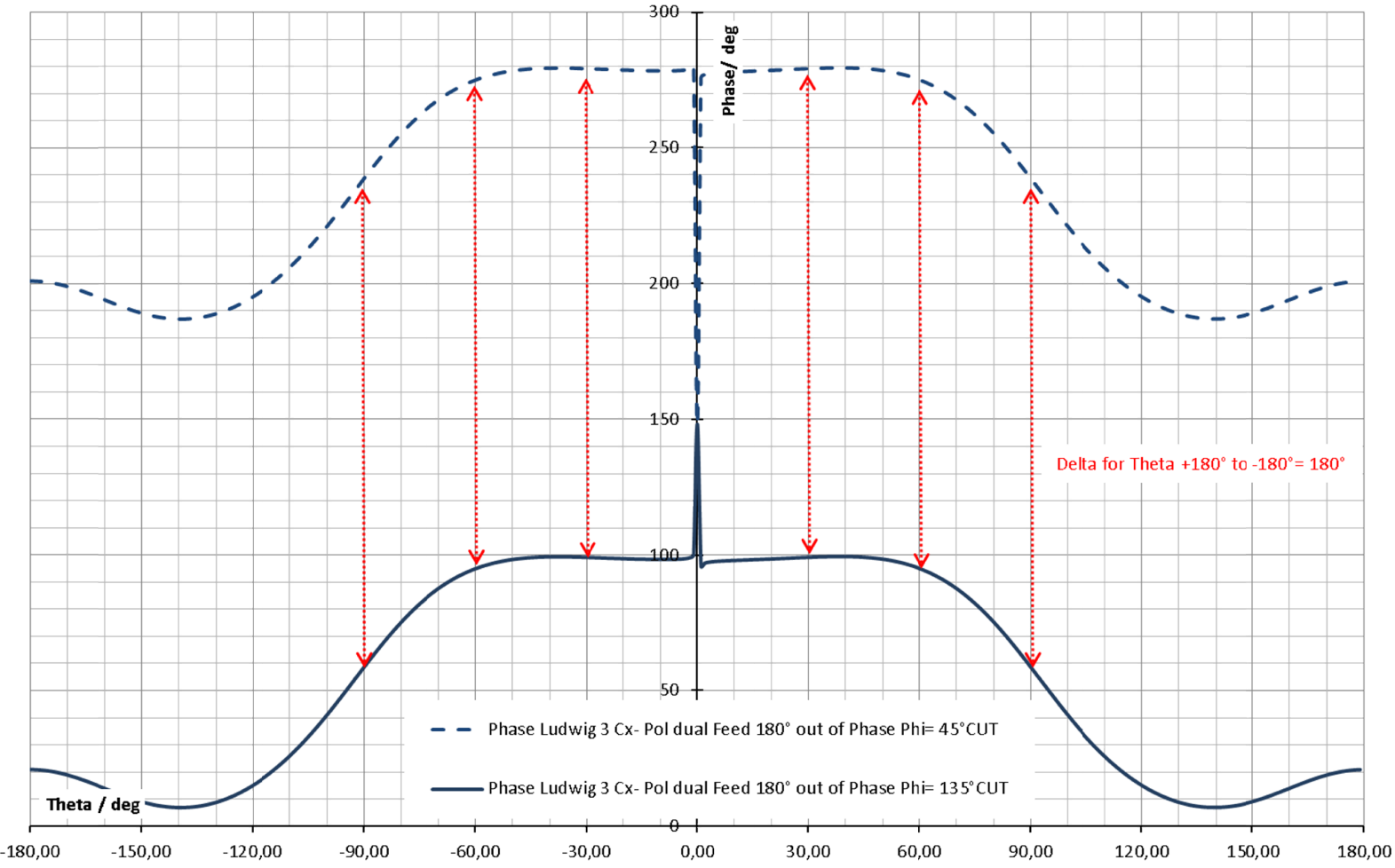

Figure 7. Phase difference of $180^{\circ}$ for $\Phi=45^{\circ}$ Cut (dashed) compared to $\Phi=135^{\circ}$ Cut, established by differential feeding technique. The $180^{\circ}$ phase difference is very useful in antenna array configurations for canceling out the x-pol components in far field from neighboring elements.

radars are working in pulse alternating or simultaneously transmitting dual polarization modes. For these dual polarization modes the single fed antenna will be equipped with two feeds; one for horizontal and one for vertical polarization. The differential fed patch antenna accommodates four feeds for dual polarization mode; two probe feeds $180^{\circ}$ out of phase for horizontal polarization and two probe feeds $180^{\circ}$ out of phase for vertical polarization. The differential fed patch antenna in dual polarization design is graphed in Fig. 8 . The isolation and S11 results from CST MWS 3-D full wave simulations are visualized in Fig. 9. It can be recognized that the differential fed dual polarized patch antenna maximum port isolation provides on desired frequency at $9.395 \mathrm{GHz}$, meanwhile the single fed dual polarized antenna very poor isolation characteristics established. The excellent isolation characteristic of the differential fed dual polarized patch antenna can be explained with Fig. 8. Let us consider an input signal at the vertical port. The signal travels through the TJunction splitter (red point in Fig. 8) and excites at first the Feed_V_1 and subsequently the Feed_V_2 with $180^{\circ}$ phase shift. Both signals exciting the patch antenna to radiate, but small signal parts (dashed lines in Fig. 8) are received by the horizontal ports Feed_H_1 and Feed_H_2. Current probes have shown from simulations that the two signals received by Feed_H_1 and Feed_H_2 are in-phase at the probe feeds

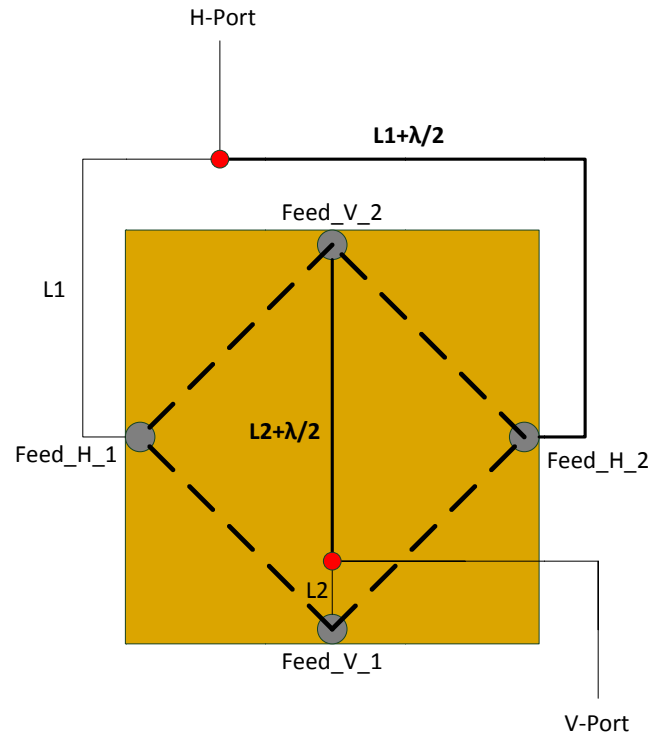

Figure 8. Graphical description for excellent isolation functionality of dual polarized patch antenna with differential feeding.

and cancel each other out at the T-Junction splitter in front of the horizontal splitter due to the length difference of $\lambda / 2$. 
It turns out, that the differential fed patch antennas with $180^{\circ}$ out of phase excitation are suitable for phased array weather radar applications and many valuable advantages compared to single fed patch antennas are evidenced and first time documented within this publication.

\section{Analysis of potential x-pol sources in microstrip patch antenna design}

Open via holes, rectangular patch edges and the position of connector and matching circuits below the shielding ground are investigated and verified as potential x-pol sources. Additionally, circular and square patch geometries are compared and variations of the patch sizes are analyzed in their x-pol performances.

\subsection{Probe fed patches with plugged via holes}

In Fig. 10a the cross section of the hole plugged probe feed is graphed. After drilling the via holes into the multilayer substrate the walls of the holes are galvanized with copper to connect the upper and lower RF layer. The holes are plugged using the following two-step process: first the via hole is filled with cured resin to generate a plane surface on both sides and secondly an additional galvanization step takes place to close the via hole and to establish a plane surface for the radiation patch antenna element and the microstrip line. From the hole plugging process one would assume that the probe feed produces lower $\mathrm{x}$-pol radiation because of plane patch antenna surface and lower resistance in the area of the feed, but CST MWS simulations have shown that the CPS is exactly (within $\pm 0.01 \mathrm{~dB}$ ) the same compared to usual open probe feeds. It was verified that hole plugging does not improve the CPS of microstrip patch antennas and the expenses for the additional plugging process can be saved.

\subsection{Bended square patch antenna edges}

By analyzing the x-pol radiation pattern in Fig. 3a-3, b-3, two (for single fed), respectively four (for differential fed) maxima above the patch edges are observable. It is assumed that $\mathrm{x}$-pol radiation contributions are generated by the four $90^{\circ}$ angles from the patch edges. Performed CST MWS simulations have evidenced that the patch antenna edge bending does not reduce the x-pol radiation. Only the resonance frequency will be shifted due to the change in absolute length of the radiating edge. After impedance re-matching of the bended patch antenna element almost the same $\mathrm{x}$-pol values with the difference of only $0.04 \mathrm{~dB}$ was observed. Edge bending does not improve the CPS and the four $90^{\circ}$ angles are not contributing significantly to the $\mathrm{x}$-pol radiation.

\subsection{CPS comparison of circular and square shaped microstrip patch antenna elements}

An intensive literature search did not reveal any adequate reference comparing the CPS of circular and square shaped microstrip patch radiators. Therefore, a comparison of the two patch antenna shapes with respect to their x-pol performances is provided below. The comparison is based on the same resonance frequency, same layer configuration, slightly different parallel stub configuration due to the different patch impedances and the same differential feeding technique.

The circular patch radiator has a diameter of $12.67 \mathrm{~mm}$, the probe via diameter is $0.4 \mathrm{~mm}$ and the probe via to edge distance is $0.5 \mathrm{~mm}$. The diameter of the isolation circle inside the shielding ground for the probe feed transition is again $0.91 \mathrm{~mm}$. The resonance frequency is located at $9.395 \mathrm{GHz}$ with a VSWR $1: 2$ bandwidth of $152 \mathrm{MHz}$. In Fig. 11a the "realized gain" of $6.66 \mathrm{dBi}$ can be recognized and the x-pol pattern from Fig. 11b shows the same results featuring four maxima comparable to Fig. 3b-3 due to the differential feeding technique. The gain is higher compared to the square patch but only due to the larger antenna patch area. The integrated CPS of $14 \mathrm{~dB}$ for differential fed circular patch antennas was established at $\pm 60^{\circ}$ azimuth position, which is almost equal to the $14.2 \mathrm{~dB}$ for differential fed square patch antennas from Fig. 4. By applying longer optimization iterations for impedance matching, the same integrated CPS for square and circular patch antenna geometries can be expected. It should be noted here that the value of $14 \mathrm{~dB}$ anyhow reflects the theoretical maximum of CPS at $\pm 60^{\circ}$ for microstrip patch antennas.

\subsection{Analysis of probe feed and its matching network position below the shielding GND of the patch antenna}

Typically, for impedance matching the probe feed positions are changed along the $y$ axis. The maximum impedance is determined by the position of the probe feed at the edge of the patch. The minimum available impedance is zero and is located at the center of the patch antenna element. For phased array application with high $\mathrm{x}$-pol requirements the differential feeding design verified in Sect. 2.2. should be applied. Consequently, only edge feeding can be realized to inject two separated signals with $180^{\circ}$ out of phase, especially for dual polarization applications. Straight connections of the edge probe feeds are seldom possible due to the limited space for feeding networks, especially for dual polarization capabilities. In this analysis the direction of the probe feeds and its matching network below the shielding ground is changed with a rotation of $90^{\circ}$ (Fig. 12a) and analyzed for X-pol (Fig. 12b) degradation.

For the differential fed square-patch with $90^{\circ}$ rotated connectors an integrated CPS of only $6.2 \mathrm{~dB}$ was observed at $\pm 60^{\circ}$ azimuth position for the $45^{\circ}$ Cut of Fig. 12, which 


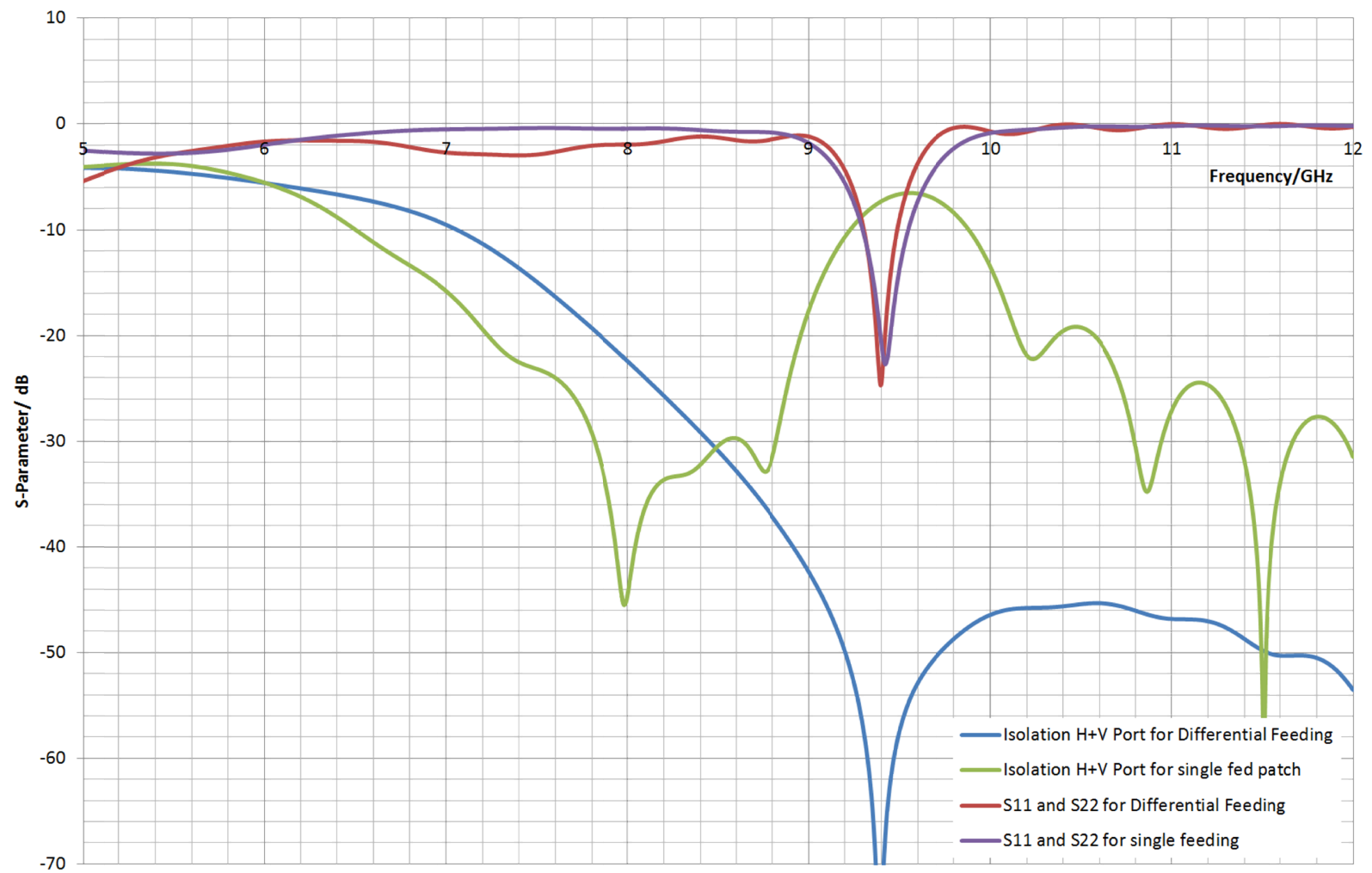

Figure 9. Comparison of port isolation characteristics from differential and single fed patch elements.

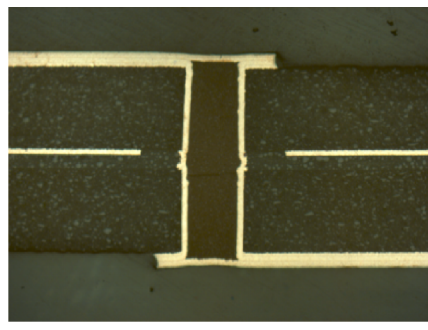

a)

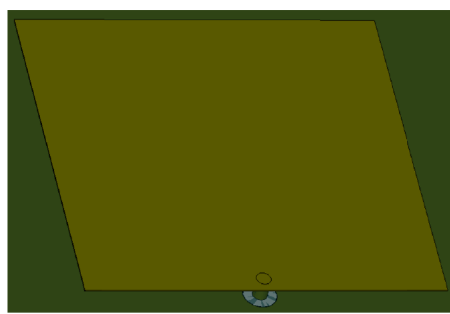

b)

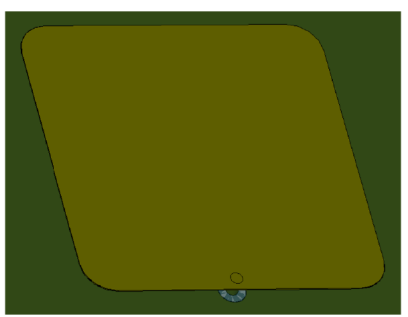

c)

Figure 10. (a) Via filled with fossil resin and closed with cooper; square patches (b) without and (c) with edge bending.

is no longer comparable to the $14.2 \mathrm{~dB}$ for differential fed square patch antennas of Fig. 4, where the probe feeds are straightly oriented (see Fig. 3b-1) and all mirror symmetries are maintained. From the not shown co-pol plot, an undesired main beam drift can also be recognized. The reason for the $\mathrm{x}$-pol degradation is probably related to the asymmetric current distribution on the shielding ground below the patch. The asymmetric current distribution on the shielding ground is generated by the matching stub positions and the current disturbances during excitation process of the antenna. This source of $\mathrm{x}$-pol radiation should be considered by every antenna designer for array designs with high $\mathrm{x}$-pol requirements.

\subsection{Analysis of antenna patch size}

Surprisingly no capable reference could be found in literature where the patch antenna size was analyzed for improved $\mathrm{x}$ pol pattern. For this reason the differential fed patch antenna design from Fig. 1c was investigated in this regard. Figure 13 shows graphically the results of the co- and x-pol pattern for variable patch sizes from 10.32 to $10.80 \mathrm{~mm}$. Table 1 summarizes all important design parameters, the results of realized gain at broadside direction and the CPS at $\Theta= \pm 60^{\circ}$.

The design example shows CPS of up to $15.50 \mathrm{~dB}$ at $\Theta= \pm 60^{\circ}$. On the other hand the realized gain stays almost constant between 5.9 and $6.0 \mathrm{dBi}$. In Fig. 13 the redistribution of the x-pol pattern for increased patch sizes becomes visible. Inside the dashed circles the CPS will be improved, 


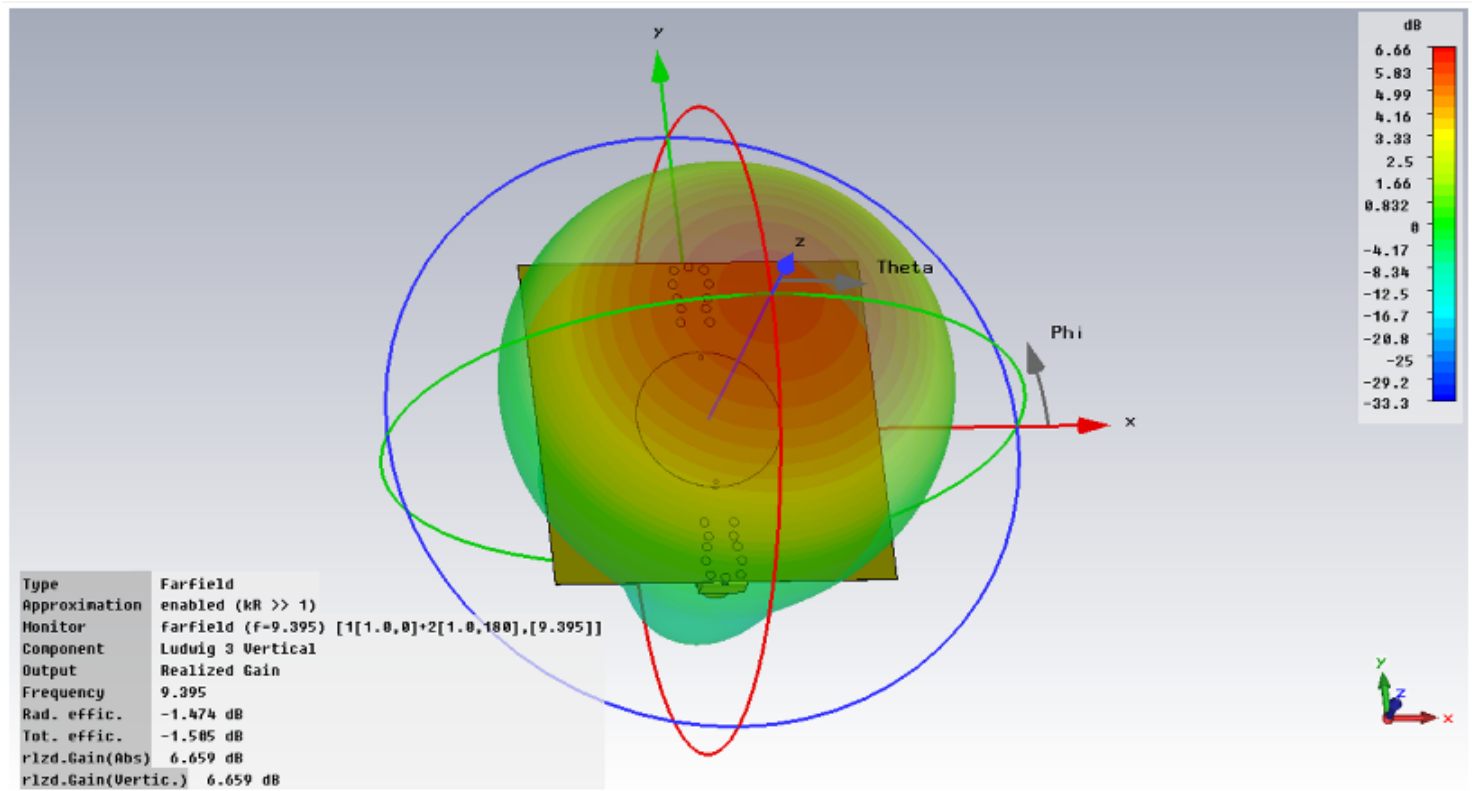

a)

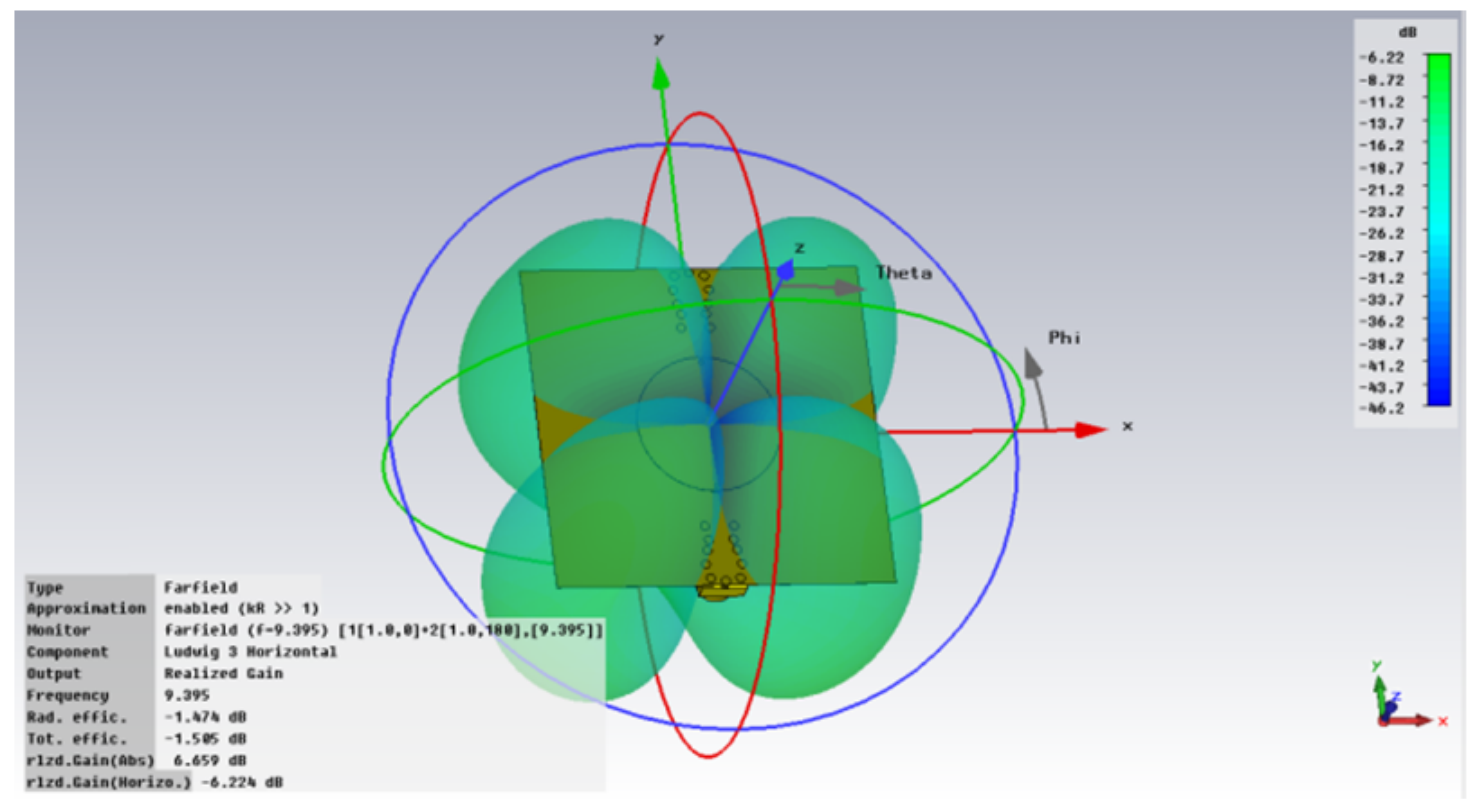

b)

\section{a) Co-pol pattern, b) $x$-pol pattern of circular shaped $X$-Band patch antenna}

Figure 11. (a) Co-pol pattern, (b) X-pol pattern of circular shaped X-Band patch antenna.

meanwhile the dashed ellipses in upper right and left corner are showing CPS degradation. This redistribution is acceptable desirable, since the CPS at invisible space behind the antenna is not that important.

The improvement of the CPS by increasing the microstrip antenna patch size is significant and can be identified as valu- able tool for antenna designers to reduce the cross polarization of microstrip patch antennas. The $\mathrm{x}$-pol radiation is moved from the center to the backside of the antenna. Especially for phased array applications the x-pol improvement for intended scan ranges between -90 and $+90^{\circ}$ is remarkable. 
Table 1. Design parameter and CPS results for variable patch sizes.

\begin{tabular}{rrrrrrrrr}
\hline $\begin{array}{r}\text { Patch } \\
\text { size }\end{array}$ & $\begin{array}{r}\text { Via } \\
\text { diameter }\end{array}$ & $\begin{array}{r}\text { GND Isolation } \\
\text { diameter }\end{array}$ & $\begin{array}{r}\text { Stub } \\
\text { width }\end{array}$ & $\begin{array}{r}\text { Stub } \\
\text { length }\end{array}$ & $\begin{array}{r}\text { Via distance } \\
\text { to patch edge }\end{array}$ & $\begin{array}{r}\text { CPS at } \\
\Theta=60^{\circ}\end{array}$ & $\begin{array}{r}\text { Realized } \\
\text { length }\end{array}$ & GND \\
\hline $\mathrm{mm}$ & $\mathrm{mm}$ & $\mathrm{mm}$ & $\mathrm{mm}$ & $\mathrm{mm}$ & $\mathrm{mm}$ & $\mathrm{dB}$ & $\mathrm{dBi}$ & $\mathrm{mm}$ \\
\hline 10.32 & 0.2 & 0.91 & 1.47 & 4.78 & 0.42 & 13.20 & 6.00 & $30 \times 30$ \\
10.55 & 0.4 & 0.91 & 1.31 & 5.13 & 0.42 & 14.20 & 5.95 & $30 \times 30$ \\
10.66 & 0.5 & 1.2 & 1.42 & 5.19 & 0.42 & 14.75 & 5.94 & $30 \times 30$ \\
10.80 & 0.7 & 1.5 & 1.49 & 5.39 & 0.50 & 15.50 & 5.90 & $30 \times 30$ \\
10.66 & 0.7 & 1.2 & 1.42 & 5.19 & 0.42 & 18.34 & 5.31 & $40 \times 40$ \\
\hline
\end{tabular}

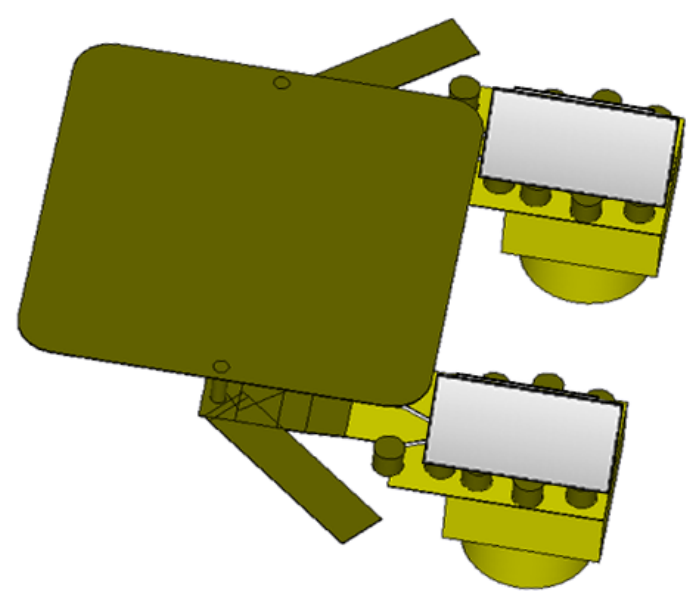

a)

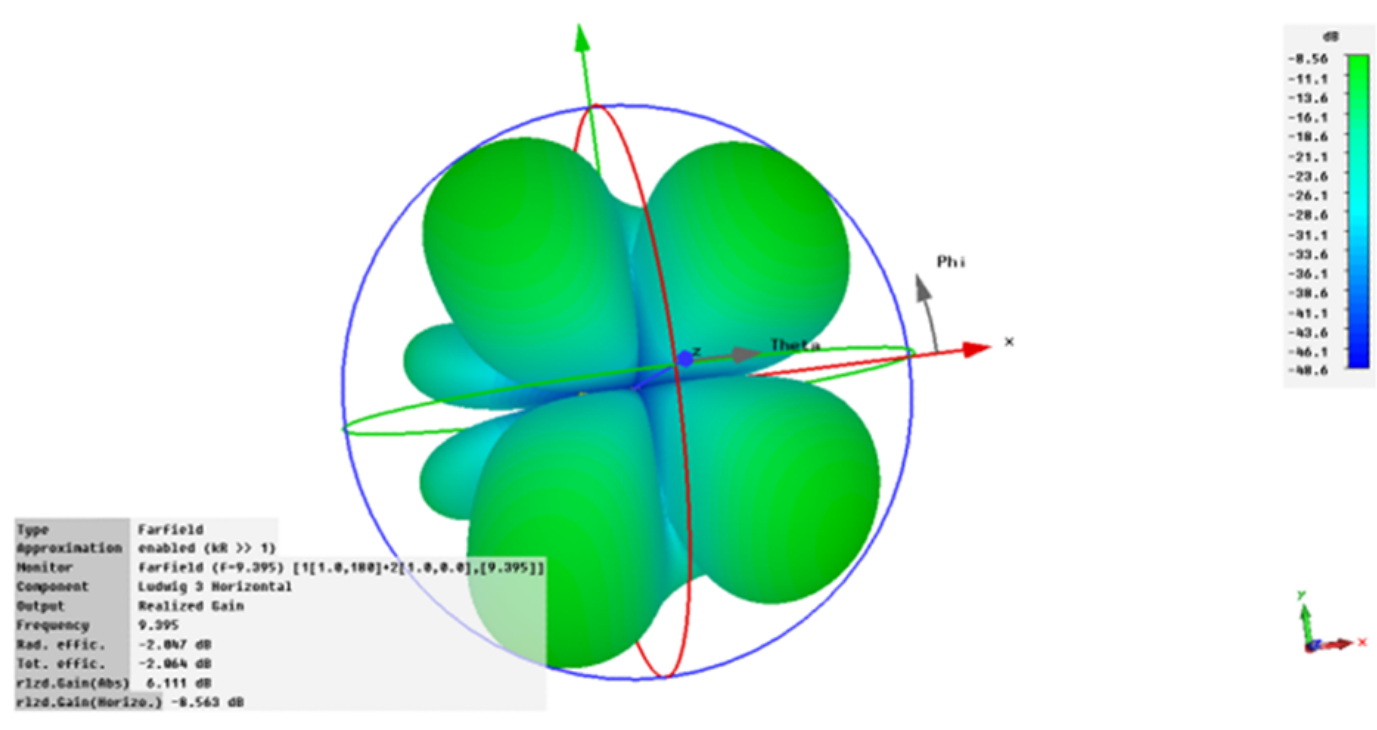

b)

Figure 12. (a) Connectors and matching stubs below the patch and shielding ground with $90^{\circ}$ rotated, (b) degraded x-pol pattern of the respective patch antenna. 


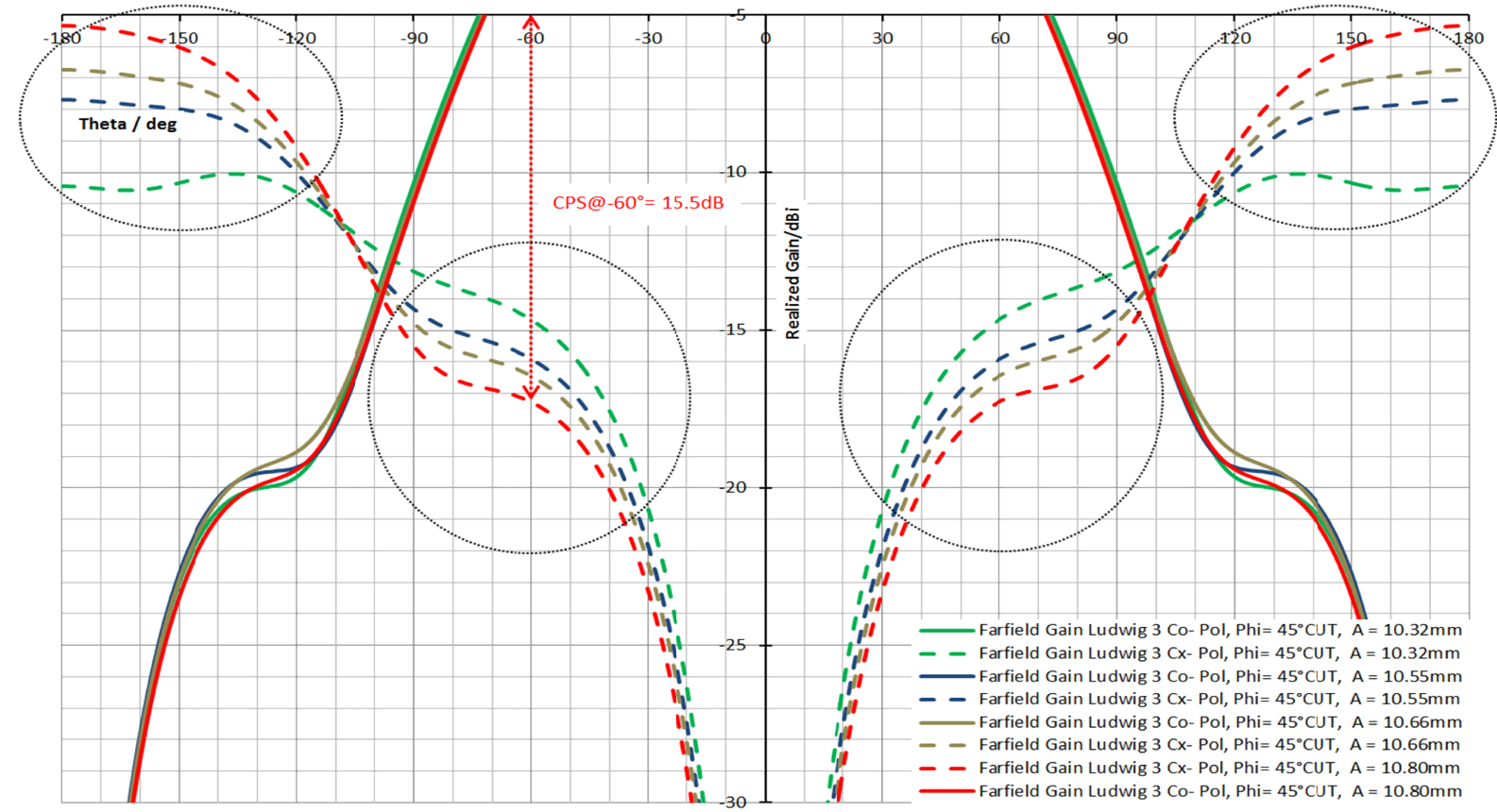

Figure 13. Analysis of co- and $\mathrm{x}$-pol radiation pattern for differential fed patch antennas with variable patch length. The patch length varies from 10.32 to $10.80 \mathrm{~mm}$. A valuable CPS improvement can be recognized in the circles between $\Theta=90$ and $30^{\circ}$. The ellipses between 180 and $120^{\circ}$ show a degradation in CPS, which is acceptable since the CPS at invisible space behind the antenna is not that important.

In a separate experiment the GND layer of the $10.66 \mathrm{~mm}$ patch design was extended from $30 \times 30$ to $40 \times 40 \mathrm{~mm}$. The realized gain in broadside direction dropped to $5.3 \mathrm{dBi}$ but the CPS at $\Theta= \pm 60^{\circ}$ became $18.34 \mathrm{~dB}$. This astonishing result is interesting for single antenna applications and their designers but is not less important for phased array applications where the GND layer and the patch to patch distance can mostly not be extended due to the occurrence of entering grating lobes.

From the results in Table 1 an empirical equation can be constructed for the length $L_{\mathrm{CPS}}^{\mathrm{opt}}$ of a quadratic patch antenna element with optimized x-pol performance:

$$
L_{\mathrm{CPS}}^{\mathrm{opt}}=K \frac{\lambda}{2 \sqrt{\varepsilon_{r}^{\mathrm{eff}}}}=1.12 \frac{\lambda}{2 \sqrt{\frac{\varepsilon_{r}+1}{2}+\frac{\varepsilon_{r}-1}{2}\left[\frac{1}{1+\sqrt{1+\frac{12 \mathrm{~h}}{W}}}\right]}} .
$$

For patch antennas working at the dominant TM010, the edge to edge distance is typically $\frac{\lambda}{2}$. The $\mathrm{x}$-pol pattern will be optimized if the patch size $L$ is slightly enlarged with $K=1.12$. The effective permittivity $\varepsilon_{r}^{\text {eff }}$ for microstrip lines where the conductor width is larger than the substrate height $(w>h)$ was found in (Hartley, 2014) and represents the part below the square root in Eq. (2).

\section{Understanding the generation of $x$-pol for microstrip patch antennas by using the new visual based field line and surface current distribution approach}

\subsection{Cavity model from literature}

In several antenna literature references e.g. Balanis (2005) or Grag et al. (2001), the microstrip patch antenna radiation process is explained by the well-known cavity model. The field distribution below the patch as illustrated in Fig. 14a generates the co- and $\mathrm{x}$-pol pattern by assuming two radiating slots with distance $L$. The uniform field distribution over the width $W$ and the cosine distribution over the length $L$ for the dominant TM010 mode can be recognized. The current and charge distribution of microstrip patch antennas is presented in Fig. 14b. Fringing fields from the cosine distribution along the length $L$ are not considered by the cavity model, due to the assumption of perfect magnetic walls surrounding the volume. Accordingly, this is one source of x-pol radiation only visible in 3-D full wave analysis.

Bhardwaj and Rahmat-Samii (2014) pointed out that the $\mathrm{X}$-pol pattern from the cavity model does not agree with the $\mathrm{x}$-pol pattern from measurements or 3-D full wave simulations. Their simulation and measurement results from single fed antennas have only shown a single null in E Cut and the cavity model predict nulls in $\mathrm{E}$ and $\mathrm{H}$ Cut.

Here another interpretation is proposed. If the field vectors from Fig. $14 \mathrm{a}$ are exactly the same in intensity and $180^{\circ}$ out 

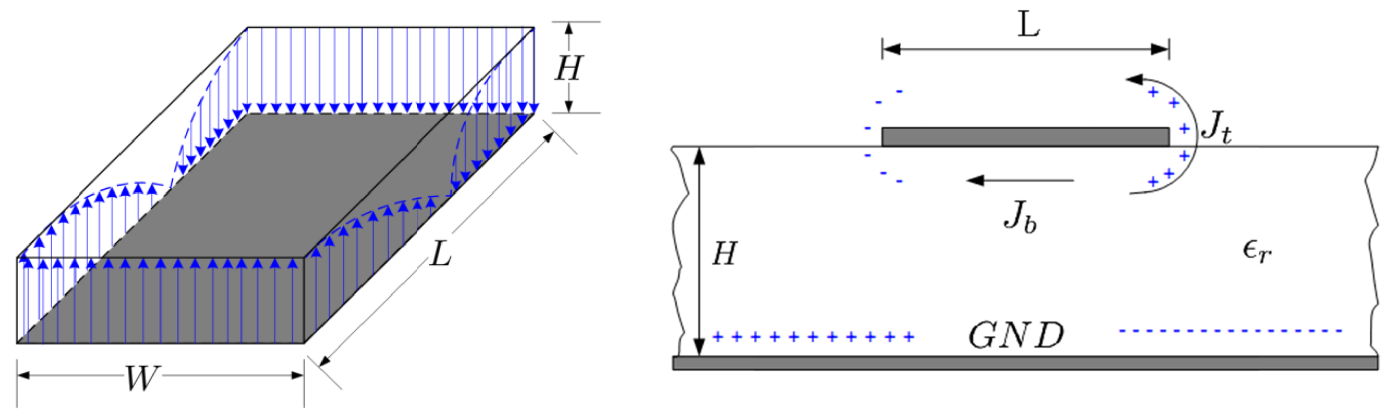

Figure 14. (a) Electrical field distribution below microstrip patch antenna with surrounding, perfect magnetic walls, (b) current and charge distribution of microstrip patch antenna (Grag, 2001).
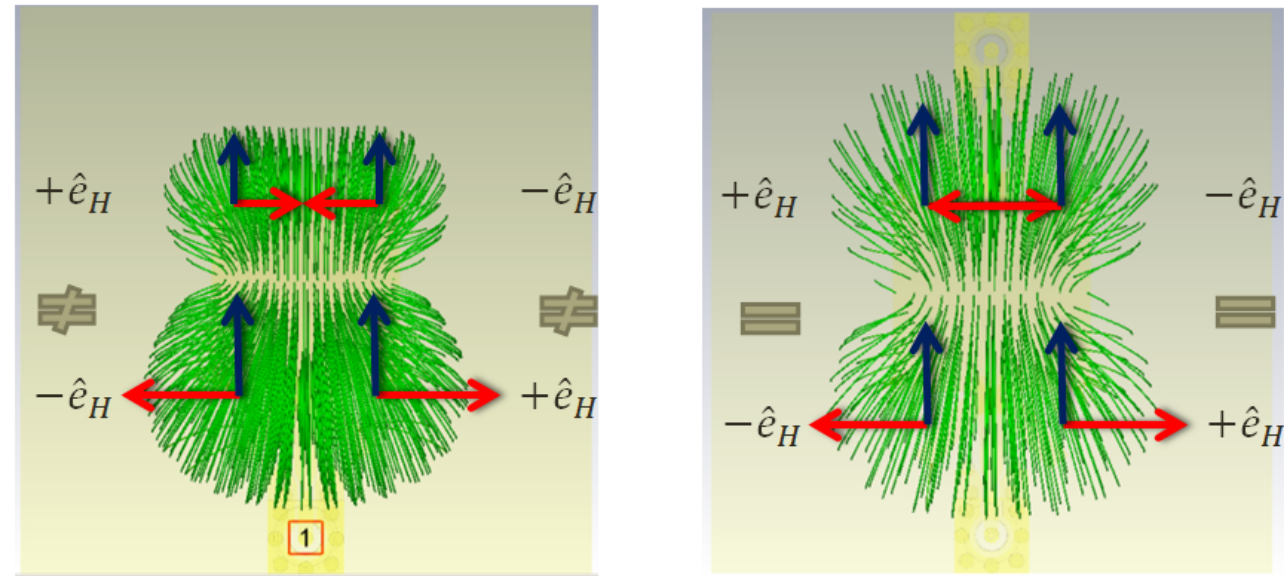

Figure 15. Field-line distributions: (a) single probe fed, (b) differential probe fed patch.

of phase at the two radiating edges, the $\mathrm{E}$ and $\mathrm{H}$ Cut can be established with two nulls like predicted from cavity model. Here the electrical field vectors have the same amplitude and show $180^{\circ}$ phase difference, indicated by the up and down direction of the electrical field vectors from Fig. 14a. On the contrary, if a patch antenna will be fed by a single probe feed like in Fig. 3a-1 the field intensities across the length $L$ are unequal (see also Fig. 15), so that a x-pol pattern with only one null in E Cut as illustrated in Fig. 3a-3 will be generated. From both figures it is visible that the $\mathrm{x}$-pol contribution is much higher on the radiating edge side, where the probe feed is located. This effect will now be analyzed and discussed in detail by the field line and surface current distribution approach in the following Sects. 4.2 and 4.3.

\subsection{Visual based field line distribution approach for $\mathrm{x}$-pol explanation}

The field line approach uses the field line generation process from 3-D full wave analysis visualized with CST MWS. Figure 15 illustrates the field line distribution for single probe feed excitation (Fig. 15a) and differential excitation with two signals $180^{\circ}$ out of phase (Fig. 15b). As mentioned before, the field intensities below the patch, across the length $L$ is unequal for single excitation (Fig. 16a) and equal for the differential excitation (Fig. 16b). The insertion loss of the path from the single probe feed to radiating edge on the opposite side is very low and the difference in field intensity is not explained herewith. A time delay causes the field intensity differences for a given point of time. As a consequence, the field line development process on the probe feed side is at advanced stage, with respect to the opposite sided radiating edge without the feed probe. The difference in field line strength between the upper and lower half of the patch at a given point of time generates the higher $\mathrm{x}$-pol pattern and no null in $\mathrm{H}$ Cut will be produced in far field. If the X-pol contribution (generated by the field lines which are not exactly vertical (co-pol) oriented for the example in Fig. 15 of the electrical field vectors on upper and lower half of the patch) are equal in strength and $180^{\circ}$ out of phase the cancellation in far field takes place and the null in $\mathrm{H}$ Cut will be generated. From Fig. 15b one can observe a very symmetrical and time synchronized field line development process. 3-D animations of the field line development process over a complete RF phase cycle are showing that the opposite $180^{\circ}$ out of phase field lines are always synchronized in time and of 


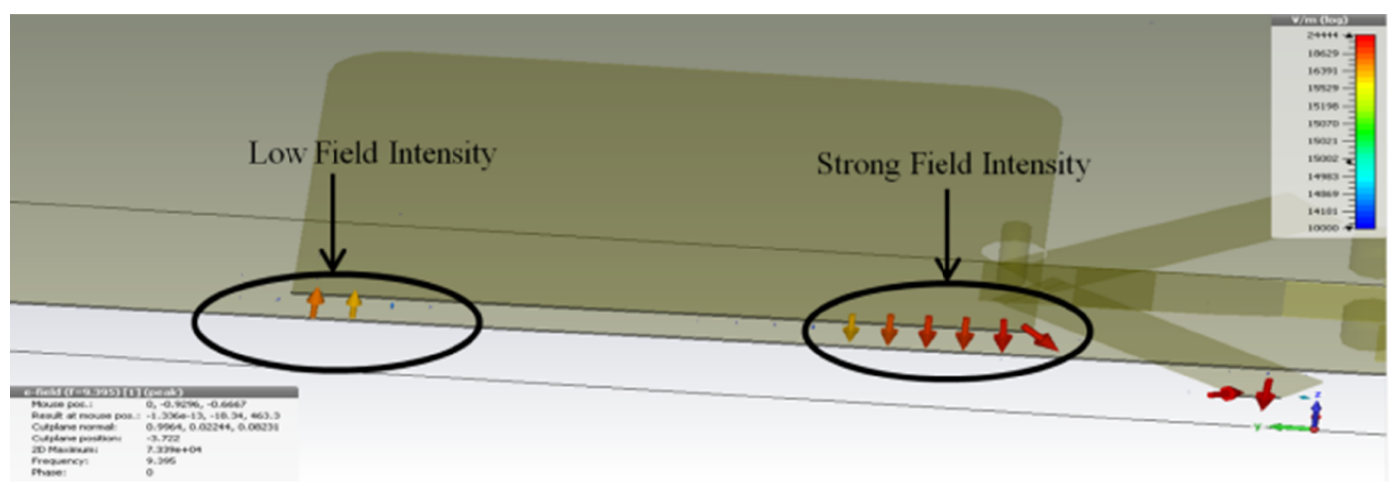

a)

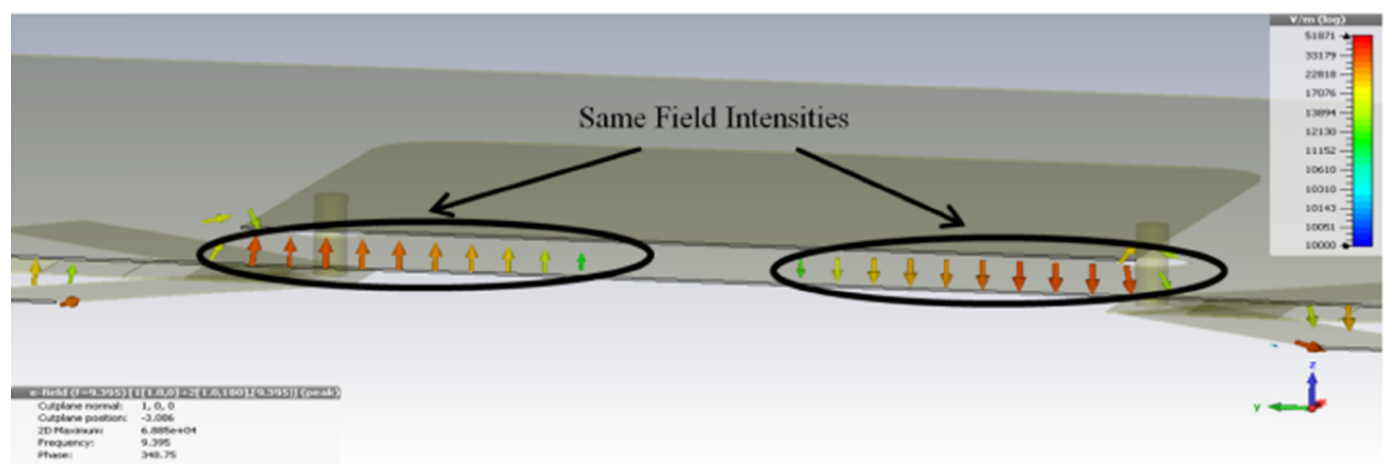

b)

Figure 16. (a) Field distribution below the patch element (a) is different in strength and phase for single feed patch antennas (b) is equal in strength and exactly $180^{\circ}$ out of phase for differential fed patch antennas.

the same intensity. By further observing Fig. 15a and b, the two and four typical x-pol maxima visible in 45 and $135^{\circ}$ Cuts can be derived. Figure $15 \mathrm{~b}$ shows that the field lines with the strongest horizontal electrical vector contribution ( $\mathrm{x}$ pol contribution) are located exactly above the 4 patch corners. Correspondingly, the very symmetric x-pol pattern with the typical four x-pol maxima over 45 and $135^{\circ}$ Cut will be generated as highlighted in Fig. 3b-4. The visualization of the field vectors helps to understand that the opposite x-pol vectors $\hat{\boldsymbol{e}}_{\mathrm{H}}$ (in red) must be the same of strength and exactly $180^{\circ}$ out of phase to cancel each other out in the antenna phase center of the H Cut. This is certainly only the case for the differential fed antenna in Fig. 15b.

\subsection{Visual based surface current distribution approach for $x$-pol explanation}

Another approach for understanding the generation process of $\mathrm{x}$-pol is the observation of surface currents from microstrip patch antennas. For this approach every single current vector from Fig. 17 can be interpreted as an elementary electric dipole (Hertzian Dipole) located on the patch antenna surface. By applying the right hand law, small dipole patterns are generated which add in the far field. As already discov- ered by the analysis of the field line approach, the mirror symmetry of field vectors is of significant importance for the reduction of $\mathrm{x}$-pol radiation. The same is true for the distribution of the currents on the patch surface. Figure $17 \mathrm{a}$ shows the surface current distribution of the single fed microstrip patch antenna. Figure $17 \mathrm{~b}$ shows the current distribution on the differential fed microstrip patch antenna, excited with two signals $180^{\circ}$ out of phase. By thoroughly investigating the current vector directions from Fig. 17a, the different vector orientations close to the two radiating edges are unambiguous. The patch surface at the feed side shows much stronger horizontally oriented $\mathrm{E}$ field vectors (x-pol). This explains the stronger $x$-pol radiation pattern values from Fig. 3a-3 and the stronger field lines in Fig. 15a. Furthermore, the current distribution on the surface of the patch is not symmetrical, so that no perfect cancellation in far field can be reached for the single feed case. On the contrary, the current vectors from Fig. $17 \mathrm{~b}$ and $\mathrm{c}$ are antipodal in direction in the region close to the two opposite probe feeds and occur with exactly the same current strength. Because of the mirror symmetry of the current distributions at the feed areas, the radiated $\mathrm{x}$-polarized electrical fields (here with horizontal content) with $180^{\circ}$ phase difference cancel each other in the far field. This explains the x-pol radiation pattern from Fig. 3b-3 


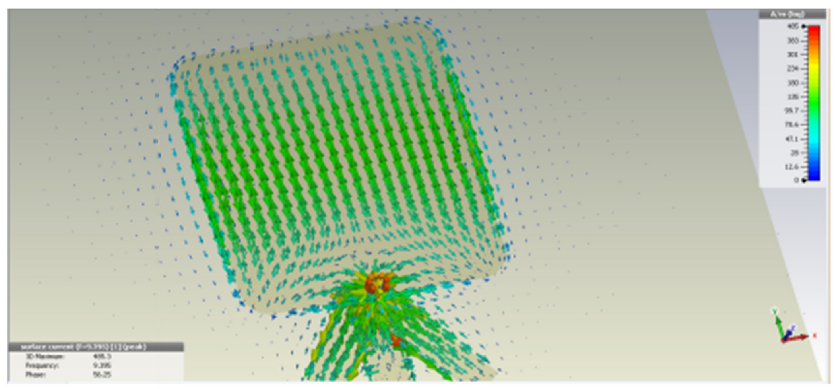

a)

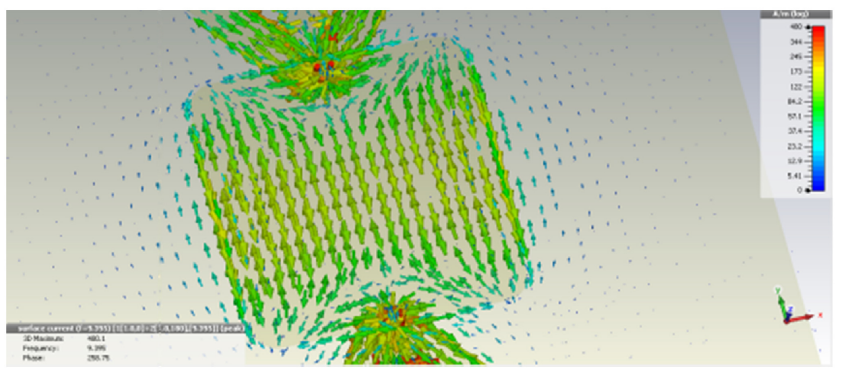

b)

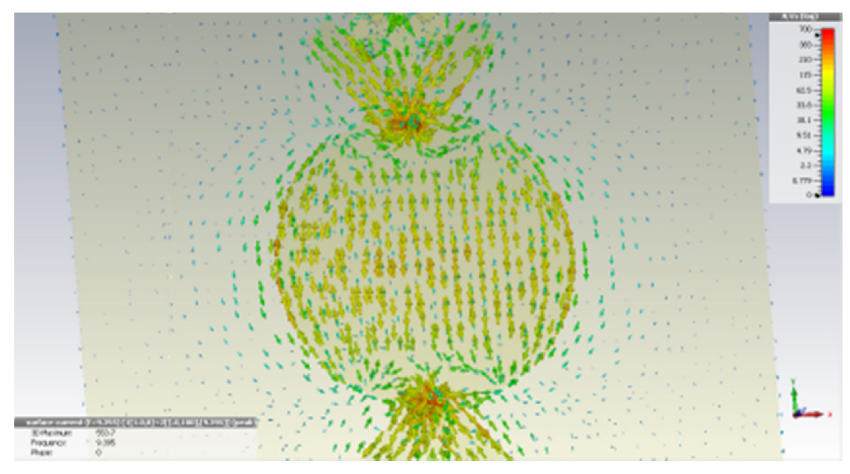

c)

Figure 17. Surface currents on (a) single fed square patch antenna; (b) differential fed square shaped patch with two signals $180^{\circ}$ out of phase (c) differential fed circular shaped patch with two signals $180^{\circ}$ out of phase.

and the symmetric field line distribution in Fig. 15b. It should be stated here, that the current distribution for circular and square patches are different. Interestingly, the different surface current distributions from circular and square patch generate almost the same CPS in far-field. Both current distributions are mirror symmetrical on the patch surfaces and the $\mathrm{x}$-pol contributions cancel in the far field.

\section{Conclusions}

The differential feeding method was verified as a valuable $\mathrm{X}$-pol reduction solution from literature research for phased array weather radar applications. Numerous feeding methods were discussed for phased array applications. The most beneficial feeding method is the probe fed antenna with its relatively small ground plane via hole transition to the radiating patches. The analysis of the geometric shape of the $\mathrm{x}$-pol pattern revealed that only $45^{\circ} / 135^{\circ}$ Cuts are able to provide sufficient information for accurate cross polarization suppression measurements, since the x-pol maxima are located on the diagonal planes. The differential feeding method was verified and compared to single probe feeding by two CST MWS modeled X-Band patch antennas. Several advantages from differential probe fed patch antennas for phased array applications were documented; the co-pol main beam becomes symmetric and the integrated CPS of $14.2 \mathrm{~dB}$ compared to only $6.2 \mathrm{~dB}$ at $\Theta= \pm 60^{\circ}$ was discovered. Furthermore, the $\mathrm{x}$-pol pattern with its four maxima and exactly $180^{\circ}$ phase difference of the two diagonal planes is advisable in order to reach reasonable $\mathrm{x}$-pol far field cancellations in antenna array configurations. The analysis of potential $\mathrm{x}$ pol sources in patch antenna element design has shown that resin filled, copper plated probe feed vias and the bending of patch edges do not improve the $\mathrm{x}$-pol radiation pattern. For the first time, circular and square shaped patch geometries were investigated and qualified as almost equal in CPS performance. The thorough investigation of the position of the probe feed and its matching network below the shielding ground of the patch has shown significant $\mathrm{x}$-pol degradation due to shielding ground current disturbances during the patch antenna radiation process. The improvement of the CPS by increasing the microstrip antenna patch size can be identified as valuable tool for antenna designers to reduce the cross polarization of microstrip patch antennas. The $\mathrm{x}$ pol radiation is moved from the center to the backside of the antenna. Especially for phased array applications the discovered x-pol improvements for intended scan ranges stated in Vollbracht (2014) is remarkable.

Starting from the discussion of the cavity model, two new visual based approaches with field line and surface current distributions were developed as a contribution for better understanding of the generation process of $\mathrm{x}$-pol radiation from microstrip patch antennas. Finally, the reason for two and four maxima in the $\mathrm{x}$-pol radiation pattern is explained with the new visual based approaches for single and differential probe fed microstrip patch antennas.

Acknowledgements. I express my gratitude to my supervisors Madhukar Chandra and Frank Gekat for the guidance and the fruitful discussions on weather radar topics and antenna theory.

Edited by: M. Chandra

Reviewed by: M. Chandra and one anonymous referee 


\section{References}

Balanis, C. A.: Antenna Theory, Analysis and Design, 3rd Edn., 1136 pp., 2005.

Bhardwaj, S. and Rahmat-Samii, Y.: Revisiting the Generation of X-pol in Rectangular Patch Antennas: A Near-Field Approach, IEEE Antenn. Propag. M., 56, 14-38, 2014.

Fulton, C. and Chappell, W.: A dual-polarized patch antenna for weather radar applications, IEEE International Conference on Microwaves, Communications, Antennas and Electronics Systems (COMCAS), Tel Aviv, Israel, 7-9 November 2011, 1-5, 2011.

Grag, R., Bhartia, P., Bahl, I., and Ittipiboon, A.: Microstrip Antenna Design Handbook, ARTECH HOUSE, London, UK, Boston, USA, 2001.

Guha, D., Biswas, M., and Antar, Y. M. M.: Microstrip Patch Antenna With Defected Ground Structure for X-pol Suppression, IEEE Antenn. Wirel. Pr, 4, 455-458, 2005.

Liang, X.-L., Zhang, Y.-M., Zhong, S.-S., and Wang, W.: Design of Dual-Polarized Microstrip Patch Antenna With Excellent Polarization Purity, Proc. 3rd International Conference on Computational Electromagnetics and Its Applications, Beijing, China, 1-4 November 2004, 197-199, 2004.
Ludwig, A. C.: The Definition of X-pol, IEEE Transactions on Antennas and Propagation, AP-21(1), 116-119, 1973.

Mohanty, A. and Das, N. K.: Characteristics Of Printed Antennas And Arrays Covered With A Layer Of Printed Strip-Grating For Suppression Of X-Pol, International Symposium of Antennas and Propagation Society, Ann Arbor, MI, USA, 28 June-2 July, 1993.

Vollbracht, D.: System specification for dual polarized low power $\mathrm{X}$-Band weather radars using phased array technology, TPH22, International Radar Conference, Lille, France, 13-17 October, 2014.

Zhou, S.-G. and Chio, T.-H.: Dual Linear Polarization Patch Antenna Array with High Isolation and Low X-pol, International Symposium on Antennas and Propagation (APSURSI), Spokane, WA, USA, 3-8 July 2011, 588-590 2011. 